\title{
The nucleon wave function in light-front dynamics
}

\author{
V.A. Karmanov团 \\ Lebedev Physical Institute, Leninsky Prospekt 53, 117924 Moscow, Russia
}

April 16, 2018

Submitted to Nucl. Phys. A

\begin{abstract}
The general spin structure of the relativistic nucleon wave function in the $3 q$ model is found. It contains 16 spin components, in contrast to 8 ones known previously, since in a many-body system the parity conservation does not reduce the number of the components. The explicitly covariant form of the wave function automatically takes into account the relativistic spin rotations, without introducing any Melosh rotation matrices. It also reduces the calculations to the standard routine of the Dirac matrices and of the trace techniques. In examples of the proton magnetic moment and of the axial nucleon form factor, with a particular wave function, we reproduce the results of the standard approach. Calculations beyond the standard assumptions give different results.
\end{abstract}

\section{Introduction}

The light-front dynamics (LFD) is a powerful approach to the theory of relativistic composite systems. In many papers, (see, for example, [1]-[13]) it was applied to the relativistic three-body systems, and, in particular, to the nucleon in $3 q$-model (see for review [14, 15]). In the paper [8] eight spin components of the nucleon wave function were indicated. Majority of calculations was done with one of the components only, corresponding to the fully symmetric momentum independent (S-wave) spin structure.

The aim of the present paper is two-fold. First of all, we discover that eight components do not exhaust the relativistic nucleon wave function. We will find another eight components, so the total number of them is sixteen. In general, this is related to the fact

*e-mail: karmanov@sci.lebedev.ru 
known long ago [16] that in a many-body system the parity conservation does not reduce the number of the spin components. Hence, for the nucleon we get $2 \times 2 \times 2 \times 2=16$. This opportunity is absent in any two-body system and in the nonrelativistic three-body one. Technically, the extra components can be constructed since the particle four-momenta in any off-energy-shell relativistic amplitude, in particular, in the wave function, are not related by the conservation law: for the minus-projections $k_{-}=k_{0}-k_{z}$ the sum of the quark momenta $k_{1,2,3}$ and the nucleon momentum $p$ are not equal to each other: $\left(k_{1}+k_{2}+k_{3}\right)_{-} \neq p_{-}$. Hence, we have in our disposal 4 four-vectors and can construct the pseudoscalar $C_{p s}=e^{\mu \nu \rho \gamma} k_{1 \mu} k_{2 \nu} k_{3 \rho} p_{\gamma}$. Due to that, in addition to "old" eight components given in [8], we construct another eight spin structures with "wrong" P-parity (the pseudoscalar structures) and then "correct" them by multiplying by $C_{p s}$. However, this way to find the spin components is not obligatory. One can construct an equivalent set of sixteen components such that only a few of them (less than eight) contain the factor $C_{p s}$. These extra components (relative to the paper [8]) are necessary in order to represent even symmetric S-wave spin structure (initially given in c.m.-system) in arbitrary system of reference in terms of the Dirac matrices sandwiched with the spinors. In other language this corresponds to multiplying the center-of-mass wave function by the Melosh rotation matrices. If we will omit these extra components and come back to the c.m.-system, we would not reproduce our initial S-wave but again will find some extra components. In general case one should start with the wave function containing all sixteen components in any system of reference. They are forming the full basis. Their total number does not depends, of course, on the representation. Their relative magnitude is determined by dynamics.

Secondly, we will represent the nucleon wave function in the $3 q$ model in the explicitly covariant form. This will allow one to use in calculations the standard Dirac-matrices algebra and the trace techniques. In particular, we will see that there is no any need to introduce explicitly any Melosh rotation matrices: the covariant approach incorporates automatically the spin rotation effects. In examples of the proton magnetic moment and of the axial nucleon form factor, we reproduce by this way the results which in the standard approach are obtained due to averaging the Melosh matrices.

The above problems will be solved in the explicitly covariant version of LFD developed in a series of papers, starting with 117 (see for review [18]). In the standard LFD the wave functions are defined on the light-front plane $t+z=0$. In the covariant approach the wave functions are defined on the light-front plane given by the invariant equation $\omega \cdot x=0$, where $\omega$ is the four-vector with $\omega^{2}=0$. This provides all the advantages of the explicit covariance, similar to advantages of the Feynman graph techniques over the old fashioned perturbation theory. The standard approach is obtained as a particular case at $\omega=(1,0,0,-1)$. However, we would like to emphasize that this particular choice of $\omega$ does not reduce the number of the components of the nucleon wave function, it is 16 in any version of LFD.

Plan of the paper is the following. In sect. 2 we introduce special representation for the wave function and the three-dimensional variables with the simple transformation properties. In sect. 3 we construct the corresponding spin matrices with the same trans- 
formation properties. In sect. H sixteen spin structures of the nucleon wave function are found, however without taking into account any permutation symmetry. In sect. 5 we remind the permutation group properties in the three-body case. In sect. 6 we take into account the appropriate permutation symmetries of the spin structures independent of momenta. Sect. 7 is devoted to incorporating the permutation symmetry in the components depending on momenta. In sect. 8 the matrix elements of the current operator, used in the form factor calculations, are found. In sect. 9 we consider the examples of the proton magnetic moment and of the axial nucleon form factor. Section 10 contains the concluding remarks.

\section{The covariant light-front wave function}

The properties of the covariant light-front wave function are described in [18]. Here we remind some of them in the three-body case.

The light front wave function of the nucleon composed from three quarks has the form:

$$
\Phi=\Phi_{\sigma_{1} \sigma_{2} \sigma_{3}}^{\sigma}\left(k_{1}, k_{2}, k_{3}, p, \omega \tau\right),
$$

where $k_{1-3}$ and $p$ are the quark and the nucleon four-momenta, $\tau$ is a scalar parameter and $\sigma_{1-3}, \sigma$ are the quark and nucleon spin projections on $z$-axis in the corresponding frames of the rest. The four-momenta are related by the conservation law:

$$
p+\omega \tau=k_{1}+k_{2}+k_{3} \equiv \mathcal{P} .
$$

The presence in (2) of the term $\omega \tau$ just reflects the nonconservation of the minuscomponents, since for $\omega=(1,0,0,-1)$ this term contributes only to the minus-projection of this equation.

Below we will use the effective mass corresponding to the four-momentum $\mathcal{P}$ :

$$
\mathcal{M}=\sqrt{\mathcal{P}^{2}} .
$$

Under rotations and the Lorentz transformations $g$ the wave function (1) is transformed by the rotation matrices $D^{\left(\frac{1}{2}\right)}\{R\}$ depending on the different rotation operators $R$ for the particles with different momenta. Namely,

$$
\begin{aligned}
& \Phi_{\sigma_{1} \sigma_{2} \sigma_{3}}^{\sigma}\left(g k_{1}, g k_{2}, g k_{3}, g p, g \omega \tau\right)=\sum_{\sigma^{\prime} \sigma_{1}^{\prime} \sigma_{2}^{\prime} \sigma_{3}^{\prime}} D_{\sigma \sigma^{\prime}}^{\left(\frac{1}{2}\right) *}\{R[g, p]\} \\
& \times D_{\sigma_{1} \sigma_{1}^{\prime}}^{\left(\frac{1}{2}\right)}\left\{R\left[g, k_{1}\right]\right\} D_{\sigma_{2} \sigma_{2}^{\prime}}^{\left(\frac{1}{2}\right)}\left\{R\left[g, k_{2}\right]\right\} D_{\sigma_{3} \sigma_{3}^{\prime}}^{\left(\frac{1}{2}\right)}\left\{R\left[g, k_{3}\right]\right\} \Phi_{\sigma_{1}^{\prime} \sigma_{2}^{\prime} \sigma_{3}^{\prime}}^{\sigma^{\prime}}\left(k_{1}, k_{2}, k_{3}, p, \omega \tau\right),
\end{aligned}
$$

where, for example, $R[g, p]$ is the following rotation operator:

$$
R[g, p]=L^{-1}(g p) g L(p) .
$$

$L(p)$ is the Lorentz boost corresponding to the velocity $\vec{v}=\vec{p} / p_{0}$. Below, in addition to the wave function $\Phi$ transformed by (4), we will introduce another representation in 
which the wave function, denoted as $\Psi$, is transformed by one and the same rotation for all the particles. This representation is defined as (see [19]):

$$
\begin{aligned}
& \Psi_{\sigma_{1} \sigma_{2} \sigma_{3}}^{\sigma}\left(k_{1}, k_{2}, k_{3}, p, \omega \tau\right)=\sum_{\sigma^{\prime}, \sigma_{1}^{\prime}, \sigma_{2}^{\prime}, \sigma_{3}^{\prime}} D_{\sigma \sigma^{\prime}}^{\left(\frac{1}{2}\right) *}\left\{R\left[L^{-1}(\mathcal{P}), p\right]\right\} D_{\sigma_{1} \sigma_{1}^{\prime}}^{\left(\frac{1}{2}\right)}\left\{R\left[L^{-1}(\mathcal{P}), k_{1}\right]\right\} \\
& \times D_{\sigma_{2} \sigma_{2}^{\prime}}^{\left(\frac{1}{2}\right)}\left\{R\left[L^{-1}(\mathcal{P}), k_{2}\right]\right\} D_{\sigma_{3} \sigma_{3}^{\prime}}^{\left(\frac{1}{2}\right)}\left\{R\left[L^{-1}(\mathcal{P}), k_{3}\right]\right\} \Phi_{\sigma_{1}^{\prime} \sigma_{2}^{\prime} \sigma_{2}^{\prime}}^{\sigma^{\prime}}\left(k_{1}, k_{2}, k_{3}, p, \omega \tau\right)
\end{aligned}
$$

where, e.g., $R\left[L^{-1}(\mathcal{P}), p\right]$ is given by (5) with $g=L^{-1}(\mathcal{P})$. The matrix $D_{\sigma_{1} \sigma_{1}^{\prime}}^{\left(\frac{1}{2}\right)}\left\{R\left[L^{-1}(\mathcal{P}), k_{1}\right]\right\}$, appeared in the transformation (6), has the form [20]:

$$
D^{\frac{1}{2}}\left\{R\left[L^{-1}(\mathcal{P}), k_{1}\right]\right\}=\frac{\left(k_{10}+m\right)\left(\mathcal{P}_{0}+\mathcal{M}\right)-\vec{\sigma} \cdot \overrightarrow{\mathcal{P}} \vec{\sigma} \cdot \overrightarrow{k_{1}}}{\left[2\left(k_{10}+m\right)\left(\mathcal{P}_{0}+\mathcal{M}\right)\left(k_{1} \cdot \mathcal{P}+m \mathcal{M}\right)\right]^{1 / 2}}
$$

and similarly for other matrices. We will not use their explicit form below.

In this representation the wave function is transformed as [19]:

$$
\begin{aligned}
& \Psi_{\sigma_{1} \sigma_{2} \sigma_{3}}^{\sigma}\left(g k_{1}, g k_{2}, g k_{3}, g p, g \omega \tau\right)=\sum_{\sigma^{\prime}, \sigma_{1}^{\prime} \sigma_{2}^{\prime} \sigma_{3}^{\prime}} D_{\sigma \sigma^{\prime}}^{\left(\frac{1}{2}\right) *}\{R[g, \mathcal{P}]\} \\
& \times D_{\sigma_{1} \sigma_{1}^{\prime}}^{\left(\frac{1}{2}\right)}\{R[g, \mathcal{P}]\} D_{\sigma_{2} \sigma_{2}^{\prime}}^{\left(\frac{1}{2}\right)}\{R[g, \mathcal{P}]\} D_{\sigma_{3} \sigma_{3}^{\prime}}^{\left(\frac{1}{2}\right)}\{R[g, \mathcal{P}]\} \Psi_{\sigma_{1}^{\prime} \sigma_{2}^{\prime} \sigma_{3}^{\prime}}^{\sigma^{\prime}}\left(k_{1}, k_{2}, k_{3}, p, \omega \tau\right) .
\end{aligned}
$$

All the matrices $D^{\left(\frac{1}{2}\right)}\{R[g, \mathcal{P}]\}$ in (\&) depend on the same rotation operator $R[g, \mathcal{P}]$.

Instead of the four-vectors $k_{1-3}, \omega$ we introduce the three-vector variables which are constructed as follows:

$$
\begin{gathered}
\vec{q}_{i}=L^{-1}(\mathcal{P}) \vec{k}_{i}=\vec{k}_{i}-\frac{\overrightarrow{\mathcal{P}}}{\mathcal{M}}\left[k_{i 0}-\frac{\vec{k}_{i} \cdot \overrightarrow{\mathcal{P}}}{\mathcal{M}+\mathcal{P}_{0}}\right], \\
\vec{n}=L^{-1}(\mathcal{P}) \vec{\omega} /\left|L^{-1}(\mathcal{P}) \vec{\omega}\right|,
\end{gathered}
$$

$i=1,2,3$. Note that $\vec{q}_{1}+\vec{q}_{2}+\vec{q}_{3}=0$. These variables are transformed by the same rotation operator $R[g, \mathcal{P}]$ which transforms the spin projections in (8):

$$
\vec{q}_{i}^{\prime}=R[g, \mathcal{P}] \vec{q}_{i}, \quad \vec{n}^{\prime}=R[g, \mathcal{P}] \vec{n} .
$$

The same situation takes place in the nonrelativistic case: all the spins and momenta are transformed by one and the same rotation. Therefore in the representation (6) and in the variables (9), (10) the problem of constructing the general form of the nucleon wave function is analogous to the nonrelativistic one. This fact simplifies very much the construction of the spin states. The only difference is the presence of the extra vector $\vec{n}$, due to dependence of the wave function on the orientation of the light-front plane. We will need also the spin operators which are transformed similarly to $\overrightarrow{q_{i}}, \vec{n}$, eq. (11). These operators will be constructed in the next section.

The transformation (6) is similar to the Melosh transformation [21]. However, the wave function $\Phi_{\sigma_{1} \sigma_{2} \sigma_{3}}^{\sigma}$, eq. (面), does not require any Melosh matrices. It can be constructed 
in terms of the Dirac spinors, that automatically provides the correct coupling of the quark spin and angular momenta in the nucleon spin. We will come back to this point below.

In the system of reference where $\overrightarrow{\mathcal{P}}=0$ the wave functions in two representations coincide with each other. Therefore, to establish the relation between the wave functions in two representations we do not need the explicit form of the rotation matrices in the above formulas. It is enough to compare the wave functions in the system where $\overrightarrow{\mathcal{P}}=0$.

\section{The three-dimensional spin matrices}

In this section we construct the three-dimensional spin matrices which $(i)$ are transformed similarly to the vectors $\vec{q}_{i}, \vec{n}$, eq. (11), and (ii) in the system where $\overrightarrow{\mathcal{P}}=0$ turn into the Pauli matrices. Then in terms of these operators and of the vectors $\vec{q}_{i}, \vec{n}$ we will construct all the spin structures of the nucleon wave function. With these matrices the problem of constructing the representations of the permutation group is also simplified very much.

The point $(i)$ is fulfilled in the representation (6), the point ( $i i)$ is provided by the projection operators constructed below.

We introduce the spinor $\bar{u}_{\mathcal{P}}^{\sigma_{1}}\left(k_{1}\right)$ in the representation (6):

$$
\bar{u}_{\mathcal{P}}^{\sigma_{1}}\left(k_{1}\right)=\sum_{\sigma_{1}^{\prime}} D_{\sigma_{1} \sigma_{1}^{\prime}}^{\left(\frac{1}{2}\right)}\left\{R\left[L^{-1}(\mathcal{P}), k_{1}\right]\right\} \bar{u}^{\sigma_{1}^{\prime}}\left(k_{1}\right),
$$

and similarly for other spinors. The explicit form of the spinor $\bar{u}^{\sigma_{1}}\left(k_{1}\right)$ and of the other ones is given in appendix A.

We construct also the projection operators:

$$
\Pi_{+}=\frac{\mathcal{M}+\hat{\mathcal{P}}}{2 \mathcal{M}}, \quad \Pi_{-}=\frac{\mathcal{M}-\hat{\mathcal{P}}}{2 \mathcal{M}},
$$

$\mathcal{P}$ is defined by (2), $\mathcal{M}$ is defined by (3) and $\hat{\mathcal{P}}=\gamma^{\mu} \mathcal{P}_{\mu}$. They have the properties:

$$
\Pi_{ \pm}^{2}=\Pi_{ \pm}, \quad \Pi_{+} \Pi_{-}=\Pi_{-} \Pi_{+}=0, \quad \gamma_{5} \Pi_{+}=\Pi_{-} \gamma_{5}, \quad U_{c} \Pi_{+}^{t}=\Pi_{-} U_{c}
$$

(the index $t$ means the transposition). In the system of reference where $\overrightarrow{\mathcal{P}}=0, \mathcal{P}_{0}=\mathcal{M}$, the projection operators obtain the simple form:

$$
\left.\Pi_{+}\right|_{\overrightarrow{\mathcal{P}}=0}=\frac{1}{2}\left(1+\gamma^{0}\right)=\left(\begin{array}{ll}
1 & 0 \\
0 & 0
\end{array}\right), \quad \Pi_{-\left.\right|_{\overrightarrow{\mathcal{P}}=0}}=\frac{1}{2}\left(1-\gamma^{0}\right)=\left(\begin{array}{ll}
0 & 0 \\
0 & 1
\end{array}\right) .
$$

Then the spin matrices we are looking for are constructed as follows:

$$
\begin{aligned}
\vec{\sigma}_{12} & =-c_{1} c_{2} \bar{u}_{\mathcal{P}}^{\sigma_{1}}\left(k_{1}\right) \Pi_{+} \vec{\gamma} \Pi_{-} U_{c} \bar{u}_{\mathcal{P}}^{\sigma_{2}}\left(k_{2}\right), \\
\vec{\sigma}_{3 N} & =-c_{3} c_{N} \bar{u}_{\mathcal{P}}^{\sigma_{3}}\left(k_{3}\right) \Pi_{+} \vec{\gamma} \gamma_{5} \Pi_{+} u_{\mathcal{P}}^{\sigma}(p),
\end{aligned}
$$

where $U_{c}=\gamma^{2} \gamma^{0}$ is the charge conjugation matrix,

$$
c_{1,2,3}=\frac{1}{\sqrt{\varepsilon_{q_{1,2,3}}+m}}, \quad c_{N}=\frac{1}{\sqrt{\varepsilon_{p}+M}} .
$$


The energies in (16) are defined in the system where $\overrightarrow{\mathcal{P}}=0$. They are expressed through the invariants:

$$
\varepsilon_{q_{i}}=\sqrt{{\overrightarrow{q_{i}}}^{2}+m^{2}}=\mathcal{P} \cdot k_{i} / \mathcal{M}, \quad \varepsilon_{p}=\mathcal{P} \cdot p / \mathcal{M} .
$$

In eqs. (15) the quantities $\vec{\sigma}_{12}, \vec{\sigma}_{3 N}$ are the $2 \times 2$ - matrices relative to the indices $\sigma_{1}, \sigma_{2}$ and $\sigma_{3}, \sigma$.

The matrices $\vec{\sigma}_{12}, \vec{\sigma}_{3 N}$ are transformed by (8), like all the amplitudes and the variables in the representation (6). In the system of reference where $\overrightarrow{\mathcal{P}}=0$, with the explicit form of the spinors given in appendix $\mathbb{A}$ (eqs.(111)-(113)) and with the projections operators (14), we find:

$$
\begin{array}{r}
\left.c_{1} \bar{u}\left(k_{1}\right) \Pi_{+}\right|_{\overrightarrow{\mathcal{P}}=0}=w_{1}^{\dagger}(1,0),\left.\quad c_{3} \bar{u}\left(k_{3}\right) \Pi_{+}\right|_{\overrightarrow{\mathcal{P}}=0}=w_{3}^{\dagger}(1,0), \\
\left.c_{2} \Pi_{-} U_{c} \bar{u}^{t}\left(k_{2}\right)\right|_{\overrightarrow{\mathcal{P}}=0}=\left(\begin{array}{r}
0 \\
-1
\end{array}\right) \sigma_{y} w_{2}^{*},\left.\quad c_{N} \Pi_{+} u(p)\right|_{\overrightarrow{\mathcal{P}}=0}=\left(\begin{array}{c}
1 \\
0
\end{array}\right) w_{N},
\end{array}
$$

$w$ 's are the two-components spinors. The factors $c_{i}$, eqs. (16), just cancel the factors $\sqrt{\varepsilon+m}$ in the bi-spinors.

With the explicit form of the Dirac matrices, eqs. (114), we see that at $\overrightarrow{\mathcal{P}}=0$ the operators $\vec{\sigma}_{12}, \vec{\sigma}_{3 N}$ indeed coincide with the Pauli matrices:

$$
\begin{aligned}
\left.\vec{\sigma}_{12}\right|_{\overrightarrow{\mathcal{P}}=0} & =\left(w_{1}^{\dagger} \vec{\sigma} \sigma_{y} w_{2}^{*}\right), \\
\left.\vec{\sigma}_{3 N}\right|_{\overrightarrow{\mathcal{P}}=0} & =\left(w_{3}^{\dagger} \vec{\sigma} w_{N}\right) .
\end{aligned}
$$

They correspond to coupling of spins of the pairs in the spin 1.

We also introduce the unit operators

$$
\begin{aligned}
1_{12} & =\left.c_{1} c_{2} \bar{u}_{\mathcal{P}}^{\sigma_{1}}\left(k_{1}\right) \Pi_{+} \gamma_{5} \Pi_{-} U_{c} \bar{u}_{\mathcal{P}}^{\sigma_{2}}\left(k_{2}\right)\right|_{\overrightarrow{\mathcal{P}}=0}=\left(w_{1}^{\dagger} \sigma_{y} w_{2}^{*}\right), \\
1_{3 N} & =\left.c_{3} c_{N} \bar{u}_{\mathcal{P}}^{\sigma_{3}}\left(k_{3}\right) \Pi_{+} \Pi_{+} u_{\mathcal{P}}^{\sigma}(p)\right|_{\overrightarrow{\mathcal{P}}=0}=\left(w_{3}^{\dagger} w_{N}\right)
\end{aligned}
$$

corresponding to the zero pair spins. Below for shortness we will often replace these operators by 1 without indices.

\section{The spin structure of the nucleon wave function}

As mentioned, in the variables $\vec{q}_{1}, \vec{q}_{2}, \overrightarrow{q_{3}}, \vec{n}$ the problem of finding the spin structures of the nucleon wave function coincides with the nonrelativistic one, since these variables are subjected to the rotations only. The only difference is the presence of the extra vector $\vec{n}$. Formally it coincides with the problem of decomposition in the invariant amplitudes of the reaction amplitude $1+2 \rightarrow 3+4+5$, since the wave function (1), like the amplitude of a process $1+2 \rightarrow 3+4+5$, depends on five four-vectors satisfying the conservation law (2). In general case, for the nonrelativistic reaction $1+2 \rightarrow 3+\ldots+n$ this problem was solved in the paper [16]. It was shown that starting with $n=5$ the number of structures is the product of the factors $(2 j+1)$, corresponding to spins of all the particles. This 
gives 16 for nucleon. Below we will find these 16 structures of the nucleon wave function and then take into account the requirements of the permutation group.

First of all, we have two structures independent of momenta, the unit operator and the scalar product of $\vec{\sigma}$ 's:

$$
1 \equiv 1_{12} 1_{3 N}, \quad \text { and } \quad \vec{\sigma}_{12} \cdot \vec{\sigma}_{3 N}
$$

Then we construct the tensor of the second rank

$$
\sigma_{12}^{i} \sigma_{3 N}^{j}
$$

It will be contracted with the corresponding tensors in the momentum space. The latters are constructed as follows.

We start with the set of ten tensors symmetric in the indices $i, j$ :

$$
\begin{array}{ll}
T_{11}^{i j}=q_{1 i} q_{1 j}-\frac{1}{3} \vec{q}_{1}^{2} \delta_{i j}, \quad T_{22}^{i j}=q_{2 i} q_{2 j}-\frac{1}{3} \vec{q}_{2}^{2} \delta_{i j}, \quad T_{33}^{i j}=q_{3 i} q_{3 j}-\frac{1}{3} \vec{q}_{3}^{2} \delta_{i j} ; \\
T_{12}^{i j}=q_{1 i} q_{2 j}+q_{2 i} q_{1 j}-\frac{2}{3} \vec{q}_{1} \cdot \overrightarrow{q_{2}} \delta_{i j}, & T_{13}^{i j}=q_{1 i} q_{3 j}+q_{3 i} q_{1 j}-\frac{2}{3} \overrightarrow{q_{1}} \cdot \vec{q}_{3} \delta_{i j}, \\
T_{23}^{i j}=q_{2 i} q_{3 j}+q_{3 i} q_{2 j}-\frac{2}{3} \vec{q}_{2} \cdot \vec{q}_{3} \delta_{i j} ; \\
T_{2 n}^{i j}=q_{1 i} n_{j}+n_{i} q_{1 j}-\frac{2}{3} \overrightarrow{q_{1}} \cdot \vec{n} \delta_{i j}, \quad n_{j}+n_{i} q_{2 j}-\frac{2}{3} \overrightarrow{q_{2}} \cdot \vec{n} \delta_{i j}, \\
T_{3 n}^{i j}=q_{3 i} n_{j}+n_{i} q_{3 j}-\frac{2}{3} \overrightarrow{q_{3}} \cdot \vec{n} \delta_{i j} ;
\end{array}
$$

Since $\vec{q}_{1}+\vec{q}_{2}+\vec{q}_{3}=0$, the tensors in eq. (20) are not independent from each other. We will express the nondiagonal tensors $T_{12}^{i j}, T_{13}^{i j}, T_{23}^{i j}$ through the diagonal ones. Namely:

$$
\begin{aligned}
& T_{12}=T_{33}-T_{11}-T_{22} \\
& T_{13}=T_{22}-T_{11}-T_{33} \\
& T_{23}=T_{11}-T_{22}-T_{33}
\end{aligned}
$$

The tensors $T_{1 n}, T_{2 n}, T_{3 n}$ are also related with each other:

$$
T_{1 n}+T_{2 n}+T_{3 n}=0 .
$$

Four conditions (21), (22) reduce the number of tensors down to six. However, since the symmetric traceless tensor have five components, there should be only five independent tensors and, hence, a relation between these six tensors. This relation is given in appendix $\mathrm{B}$ by eq. (115). Due to this relation we exclude the tensor $T_{n n}^{i j}$ which does not contain the relative momenta. Hence, we have five independent structures to be contracted with the spin tensor $\sigma_{12}^{i} \sigma_{3 N}^{j}$.

Then we construct four tensors, antisymmetric in the indices $i, j$ :

$$
\begin{aligned}
& A_{12}=q_{1 i} q_{2 j}-q_{1 j} q_{2 i} \\
& A_{1 n}=q_{1 i} n_{j}-q_{1 j} n_{i} \\
& A_{2 n}=q_{2 i} n_{j}-q_{2 j} n_{i} \\
& A_{3 n}=q_{3 i} n_{j}-q_{3 j} n_{i}
\end{aligned}
$$


Since $A_{1 n}+A_{2 n}+A_{3 n}=0$, there are three independent tensors. We will specify them later.

We can also construct the linear structures of the type $\vec{\sigma} \cdot\left[\vec{q}_{1} \times \vec{q}_{2}\right]$ (multiplied implicitly by the corresponding unit operator). By this way we find sixteen spin structures of the nucleon wave function:

$$
\begin{aligned}
& (1-2) \quad 1, \quad \vec{\sigma}_{12} \cdot \vec{\sigma}_{3 N} \text {; } \\
& (3-5) \quad T_{11}^{i j} \sigma_{12}^{i} \sigma_{3 N}^{j}, \quad T_{22}^{i j} \sigma_{12}^{i} \sigma_{3 N}^{j}, \quad T_{33}^{i j} \sigma_{12}^{i} \sigma_{3 N}^{j} \text {; } \\
& (6-7) \text { two structures of three: } \\
& T_{1 n}^{i j} \sigma_{12}^{i} \sigma_{3 N}^{j}, \quad T_{2 n}^{i j} \sigma_{12}^{i} \sigma_{3 N}^{j}, \quad T_{3 n}^{i j} \sigma_{12}^{i} \sigma_{3 N}^{j} \text {; } \\
& \text { (8) } \quad A_{12}^{i j} \sigma_{12}^{i} \sigma_{3 N}^{j} \text {; } \\
& (9-10) \text { two structures of three: } \\
& A_{1 n}^{i j} \sigma_{12}^{i} \sigma_{3 N}^{j}, \quad A_{2 n}^{i j} \sigma_{12}^{i} \sigma_{3 N}^{j}, \quad A_{3 n}^{i j} \sigma_{12}^{i} \sigma_{3 N}^{j} \text {; } \\
& (11-16) \quad \vec{\sigma}_{12} \cdot\left[\vec{q}_{1} \times \vec{q}_{2}\right], \quad \vec{\sigma}_{12} \cdot\left[\vec{q}_{1} \times \vec{n}\right], \quad \vec{\sigma}_{12} \cdot\left[\overrightarrow{q_{2}} \times \vec{n}\right], \\
& \vec{\sigma}_{3 N} \cdot\left[\overrightarrow{q_{1}} \times \overrightarrow{q_{2}}\right], \quad \vec{\sigma}_{3 N} \cdot\left[\overrightarrow{q_{1}} \times \vec{n}\right], \quad \vec{\sigma}_{3 N} \cdot\left[\overrightarrow{q_{2}} \times \vec{n}\right] .
\end{aligned}
$$

Instead of the structures $(8-10)$ one can construct

$$
\left(\left[\vec{\sigma}_{12} \times \vec{\sigma}_{3 N}\right] \cdot \vec{q}_{1}\right)\left(\vec{n} \cdot\left[\vec{q}_{1} \times \vec{q}_{2}\right]\right), \quad\left(\left[\vec{\sigma}_{12} \times \vec{\sigma}_{3 N}\right] \cdot \vec{q}_{2}\right)\left(\vec{n} \cdot\left[\vec{q}_{1} \times \vec{q}_{2}\right]\right), \quad\left(\left[\vec{\sigma}_{12} \times \vec{\sigma}_{3 N}\right] \cdot \vec{n}\right)\left(\vec{n} \cdot\left[\vec{q}_{2} \times \vec{q}_{2}\right]\right),
$$

but the double vector products are reduced to the linear combinations of $(8-10)$. Instead of the structures $(11-16)$ one can construct $\left(\vec{\sigma}_{12} \cdot \overrightarrow{q_{1}}\right)\left(\vec{n} \cdot\left[\overrightarrow{q_{1}} \times \overrightarrow{q_{2}}\right]\right),\left(\vec{\sigma}_{12} \cdot \overrightarrow{q_{2}}\right)\left(\vec{n} \cdot\left[\overrightarrow{q_{1}} \times \overrightarrow{q_{2}}\right]\right)$, etc., but they are linearly expressed through the structures (11-16) by the formulas (117) given in appendix C.

The structures (1-16) of (24) are forming the basis for the relativistic nucleon wave function. The coefficients in the decomposition of the wave function in terms of this basis are the scalar functions. They depend on the quark momenta $\vec{q}_{1-3}$ and on $\vec{n}$ and are found from dynamics.

In the nonrelativistic limit the wave function does not depend on the orientation of the light-front plane, hence the contribution of the components depending on $\vec{n}$ disappears (as well as the $\vec{n}$-dependence of the scalar functions). The wave function in this limit contains eight structures of eqs. (24), namely, (1-5), (8) and two $\vec{n}$-independent structures of $(11-16)$. This just corresponds to the fact that the momentum conservation in the nonrelativistic limit obtains the form: $\vec{p}=\vec{k}_{1}+\vec{k}_{2}+\vec{k}_{3}$, and in the system of the rest $\vec{p}=0$ we have only two independent three-vectors. Note that this reduction is a peculiarity of the two- and three-body systems. In a system of $n$ particles with $n>3$ the nonrelativistic limit does not decrease the number of components. For example, the wave function of ${ }^{4} \mathrm{He}$ contains 16 components both in relativistic and in nonrelativistic cases.

The basis (24) is not yet irreducible respective to the permutation group. The corresponding irreducible basis will be constructed below.

We also list all the spin structures in the four-dimensional form:

$$
\text { (1) } \quad\left[\bar{u}\left(k_{1}\right) U_{c} \bar{u}\left(k_{2}\right)\right]\left[\bar{u}\left(k_{3}\right) \gamma_{5} u(p)\right]
$$




$$
\begin{aligned}
& {\left[\bar{u}\left(k_{1}\right) \gamma_{5} U_{c} \bar{u}\left(k_{2}\right)\right]\left[\bar{u}\left(k_{3}\right) u(p)\right]} \\
& {\left[\bar{u}\left(k_{1}\right) \hat{p} \gamma_{5} U_{c} \bar{u}\left(k_{2}\right)\right]\left[\bar{u}\left(k_{3}\right) u(p)\right]} \\
& {\left[\bar{u}\left(k_{1}\right) \hat{p} U_{c} \bar{u}\left(k_{2}\right)\right]\left[\bar{u}\left(k_{3}\right) \gamma_{5} u(p)\right]} \\
& {\left[\bar{u}\left(k_{1}\right) \gamma^{\mu} U_{c} \bar{u}\left(k_{2}\right)\right]\left[\bar{u}\left(k_{3}\right) \gamma_{\mu} \gamma_{5} u(p)\right]} \\
& {\left[\bar{u}\left(k_{1}\right) \gamma^{\mu} \gamma_{5} U_{c} \bar{u}\left(k_{2}\right)\right]\left[\bar{u}\left(k_{3}\right) \gamma_{\mu} u(p)\right]} \\
& {\left[\bar{u}\left(k_{1}\right) \sigma^{\mu \nu} p_{\nu} U_{c} \bar{u}\left(k_{2}\right)\right]\left[\bar{u}\left(k_{3}\right) \gamma_{\mu} \gamma_{5} u(p)\right]} \\
& {\left[\bar{u}\left(k_{1}\right) \sigma^{\mu \nu} U_{c} \bar{u}\left(k_{2}\right)\right]\left[\bar{u}\left(k_{3}\right) \sigma_{\mu \nu} \gamma_{5} u(p)\right]} \\
& C_{p s}\left[\bar{u}\left(k_{1}\right) U_{c} \bar{u}\left(k_{2}\right)\right]\left[\bar{u}\left(k_{3}\right) u(p)\right] \\
& C_{p s}\left[\bar{u}\left(k_{1}\right) \gamma_{5} U_{c} \bar{u}\left(k_{2}\right)\right]\left[\bar{u}\left(k_{3}\right) \gamma_{5} u(p)\right] \\
& C_{p s}\left[\bar{u}\left(k_{1}\right) \hat{p} U_{c} \bar{u}\left(k_{2}\right)\right]\left[\bar{u}\left(k_{3}\right) u(p)\right] \\
& \left.C_{p s}\left[\bar{u}\left(k_{1}\right) \hat{p} \gamma_{5} U_{c} \bar{u}\left(k_{2}\right)\right]\right]\left[\bar{u}\left(k_{3}\right) \gamma_{5} u(p)\right] \\
& C_{p s}\left[\bar{u}\left(k_{1}\right) \gamma^{\mu} U_{c} \bar{u}\left(k_{2}\right)\right]\left[\bar{u}\left(k_{3}\right) \gamma_{\mu} u(p)\right] \\
& C_{p s}\left[\bar{u}\left(k_{1}\right) \gamma^{\mu} \gamma_{5} U_{c} \bar{u}\left(k_{2}\right)\right]\left[\bar{u}\left(k_{3}\right) \gamma_{\mu} \gamma_{5} u(p)\right] \\
& C_{p s}\left[\bar{u}\left(k_{1}\right) \sigma^{\mu \nu} p_{\nu} U_{c} \bar{u}\left(k_{2}\right)\right]\left[\bar{u}\left(k_{3}\right) \gamma_{\mu} u(p)\right] \\
& C_{p s}\left[\bar{u}\left(k_{1}\right) \sigma^{\mu \nu} U_{c} \bar{u}\left(k_{2}\right)\right]\left[\bar{u}\left(k_{3}\right) \sigma_{\mu \nu} u(p)\right]
\end{aligned}
$$

where $C_{p s}$ is the pseudoscalar:

$$
C_{p s}=\epsilon^{\mu \nu \rho \gamma} k_{1 \mu} k_{2 \nu} k_{3 \rho} p_{\gamma} .
$$

By means of eq. (21) $C_{p s}$ can be transformed as: $C_{p s} \propto \epsilon^{\mu \nu \rho \gamma} k_{1 \mu} k_{2 \nu} \omega_{\rho} \mathcal{P}_{\gamma}$. In the system of reference where $\overrightarrow{\mathcal{P}}=0$ it turns into $\vec{n} \cdot\left[\vec{q}_{1} \times \vec{q}_{2}\right]$.

The structures (1-8) of (25) exactly coincide with ones given in the paper [8]. The structures (9-16) of (26) are obtained from (1-8) by deleting or adding $\gamma_{5}$ and multiplying by $C_{p s}$, that does not change their parity. They disappear on the energy shell $p=$ $k_{1}+k_{2}+k_{3}$, since in this case $C_{p s}=0$.

Any other structure is expressed through these ones. For example, instead of the structures (11),(12) and (15) one can take the structures obtained from (3),(4) and (7) by the replacement $p \rightarrow k_{3}$, and reduce by this way the number of structures containing the factor $C_{p s}$.

There is no any one-to-one correspondence between a given three-dimensional structures (24) and one of the four-dimensional structures (25-26). A given structure from (25) 26) is expressed through a linear combination of (24). The increase of the number of components from 8 to 16 in comparison to the paper [8] is not only due to the $\vec{n}$-depending structures. The latters are already implicitly included in the "old" structures eq. (25). For example, the structure (1) of eq. (25) generates the $\vec{n}$-depending three-dimensional structure of the type $\left(\vec{\sigma}_{12} \cdot \vec{q}_{1}\right)\left(\vec{\sigma}_{3 N} \cdot \vec{n}\right)$, since at $\overrightarrow{\mathcal{P}}=0$ we get in the argument of the spinor $u(p): \vec{p}=-\vec{\omega} \tau \propto \vec{n}$.

The $\vec{n}$-depending components may be important. The example is given by the deuteron wave function [22], where $\vec{n}$-depending component, so called $f_{5}$, dominates starting with $q=0.5 \mathrm{GeV} / \mathrm{c}$. 
We emphasize that the extra structures $(9-16)$ in (26) are not a peculiarity of the covariant formulation of LFD. The four-vector $\omega$ even does not enter in (26). Therefore another eight structures of the nucleon wave function should appear in any light-front approach.

In the following we will transform the basis (24) to the form providing the irreducible representations of the permutation group and represent it in terms of the bi-spinors.

\section{The permutation group in the three-body case}

\subsection{The irreducible representations}

We briefly remind the general construction of the irreducible representations of the permutation group in the three-body case.

By permutations of the arguments of the function $\psi(123)$ we get six functions. Define their six linear combinations corresponding to the Young diagrams.

Fully symmetric:

$\psi_{S}=$\begin{tabular}{|l|l|l|}
\hline 1 & 2 & 3
\end{tabular}$=\psi(123)+\psi(213)+\psi(231)+\psi(321)+\psi(312)+\psi(132)$.

Fully antisymmetric:

$$
\psi_{A}=\begin{array}{|l|}
\hline 1 \\
\hline 2 \\
\hline 3 \\
\hline
\end{array}=\psi(123)-\psi(213)+\psi(231)-\psi(321)+\psi(312)-\psi(132) .
$$

Mixed symmetry doublet:

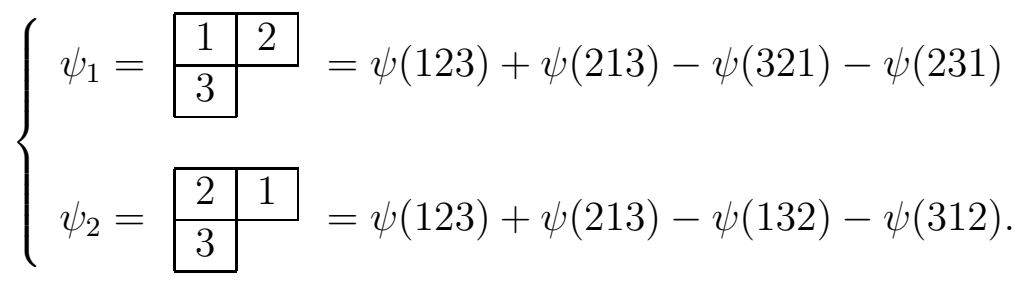

Another mixed symmetry doublet:

$$
\left\{\begin{array}{l}
\psi_{1}^{\prime \prime}=\begin{array}{l|l}
\hline 1 & 3 \\
\hline 2 & =\psi(123)+\psi(321)-\psi(213)-\psi(312)
\end{array} \\
\psi_{2}^{\prime \prime}=\begin{array}{l|l}
3 & 1 \\
\hline 2 & =\psi(123)+\psi(321)-\psi(132)-\psi(231) .
\end{array}
\end{array}\right.
$$

Relative to the permutations $P_{i j}$ of the particles $i j$ the mixed symmetry doublets are transformed as follows:

$$
\begin{aligned}
& P_{12}\left(\begin{array}{l}
\psi_{1} \\
\psi_{2}
\end{array}\right)=\left(\begin{array}{l}
\psi_{2} \\
\psi_{1}
\end{array}\right), P_{13}\left(\begin{array}{l}
\psi_{1} \\
\psi_{2}
\end{array}\right)=\left(\begin{array}{l}
-\psi_{1} \\
-\psi_{1}+\psi_{2}
\end{array}\right), P_{23}\left(\begin{array}{l}
\psi_{1} \\
\psi_{2}
\end{array}\right)=\left(\begin{array}{l}
\psi_{1}-\psi_{2} \\
-\psi_{2}
\end{array}\right) \\
& P_{12}\left(\begin{array}{c}
\psi_{1}^{\prime \prime} \\
\psi_{2}^{\prime \prime}
\end{array}\right)=\left(\begin{array}{l}
-\psi_{1}^{\prime \prime} \\
-\psi_{1}^{\prime \prime}+\psi_{2}^{\prime}
\end{array}\right), P_{13}\left(\begin{array}{c}
\psi_{1}^{\prime \prime} \\
\psi_{2}^{\prime \prime}
\end{array}\right)=\left(\begin{array}{c}
\psi_{2}^{\prime \prime} \\
\psi_{1}^{\prime \prime}
\end{array}\right), P_{23}\left(\begin{array}{c}
\psi_{1}^{\prime \prime} \\
\psi_{2}^{\prime \prime}
\end{array}\right)=\left(\begin{array}{l}
\psi_{1}^{\prime \prime}-\psi_{2}^{\prime \prime} \\
-\psi_{2}^{\prime \prime}
\end{array}\right)
\end{aligned}
$$


Instead of (31) we introduce another pair of the mixed symmetry functions:

$$
\left\{\begin{array}{l}
\psi_{1}^{\prime}=\psi_{2}^{\prime \prime}-\psi_{1}^{\prime \prime}=\psi(213)+\psi(312)-\psi(132)-\psi(231) \\
\psi_{2}^{\prime}=\psi_{2}^{\prime \prime}=\psi(123)+\psi(321)-\psi(132)-\psi(231)
\end{array}\right.
$$

Relative to permutations they are transformed identically to eq. (32). Hence, we can consider the different mixed symmetry doublets with identical transformation properties (32).

\subsection{The products of two irreducible representations}

Below we will construct the spin-isospin functions $\phi$ with a given symmetry from the products of irreducible representations formed by the spin functions $\varphi$ with a given symmetry and by the mixed symmetry isospin functions $\chi$. As well known, there are no any fully symmetric and antisymmetric isospin functions $\chi_{S}, \chi_{A}$ (see below sect. 6.1). Then from the spin-isospin functions $\phi$ and the invariant functions $\psi$, depending on momenta, we will construct the fully symmetric nucleon wave functions $\Psi$ (implicitly multiplied by the antisymmetric color singlet wave function).

We start with most general formula containing all possible products of $\varphi$ 's and $\chi$ 's:

$$
\left\{\begin{array}{l}
\phi_{1}=a_{1} \varphi_{1} \chi_{1}+b_{1} \varphi_{2} \chi_{2}+c_{1} \varphi_{1} \chi_{2}+d_{1} \varphi_{2} \chi_{1} \\
\phi_{2}=a_{2} \varphi_{1} \chi_{1}+b_{2} \varphi_{2} \chi_{2}+c_{2} \varphi_{1} \chi_{2}+d_{2} \varphi_{2} \chi_{1}
\end{array}\right.
$$

where $\varphi_{1,2}$ and $\chi_{1,2}$ satisfy (32). We also consider the similar formulas with $\varphi$ fully symmetric and antisymmetric. We find the coefficients in (35) from the conditions that these $\phi_{1}, \phi_{2}$ were fully symmetric, antisymmetric or also satisfy (32).

For the fully symmetric function $\phi_{S}$ we find:

$$
\phi_{S}=2\left(\varphi_{1} \chi_{1}+\varphi_{2} \chi_{2}\right)-\varphi_{1} \chi_{2}-\varphi_{2} \chi_{1}
$$

The fully antisymmetric function reads:

$$
\phi_{A}=\varphi_{1} \chi_{2}-\varphi_{2} \chi_{1}
$$

For the mixed symmetry functions we obtain:

$$
\left\{\begin{array} { l } 
{ \phi _ { 1 } = \varphi _ { S } \chi _ { 1 } } \\
{ \phi _ { 2 } = \varphi _ { S } \chi _ { 2 } }
\end{array} ( a ) \quad \left\{\begin{array} { l } 
{ \phi _ { 1 } = \varphi _ { A } ( \chi _ { 1 } - 2 \chi _ { 2 } ) } \\
{ \phi _ { 2 } = \varphi _ { A } ( 2 \chi _ { 1 } - \chi _ { 2 } ) }
\end{array} ( b ) \quad \left\{\begin{array}{l}
\phi_{1}=\varphi_{1}\left(\chi_{1}-\chi_{2}\right)-\varphi_{2} \chi_{1} \\
\phi_{2}=-\varphi_{1} \chi_{2}+\varphi_{2}\left(\chi_{2}-\chi_{1}\right)
\end{array}(c)\right.\right.\right.
$$

By this way we find for the total fully symmetric nucleon wave function:

$$
\begin{aligned}
& \Psi_{S}=\psi_{S} \phi_{S} \\
& \Psi_{S}=\psi_{A} \phi_{A} \\
& \Psi_{S}=2\left(\psi_{1} \phi_{1}+\psi_{2} \phi_{2}\right)-\psi_{1} \phi_{2}-\psi_{2} \phi_{1}
\end{aligned}
$$

One should substitute in (41) the different mixed symmetry doublets $\phi_{1,2}$ from (38). 


\section{The components independent of momenta}

\subsection{The spin-isospin functions with a given symmetry}

To establish the permutation properties, we put $\overrightarrow{\mathcal{P}}=0$ and denote the spin functions (1-2) of eq. (24), corresponding to the spins of pairs 0 and 1, as:

$$
\varphi^{(0)} \equiv \varphi^{(0)}(123)=\left(w_{1}^{\dagger} \sigma_{y} w_{2}^{*}\right)\left(w_{3}^{\dagger} w_{N}\right), \quad \varphi^{(1)} \equiv \varphi^{(1)}(123)=\left(w_{1}^{\dagger} \vec{\sigma} \sigma_{y} w_{2}^{*}\right)\left(w_{3}^{\dagger} \vec{\sigma} w_{N}\right) .
$$

We keep the order of the quark spinors 123. Using the Fierz identities, given in appendix D, one can establish the following permutation properties:

$$
\begin{aligned}
\varphi^{(0)}(123)=-\varphi^{(0)}(213)=\varphi^{(0)}, \quad \varphi^{(0)}(321) & =-\varphi^{(0)}(231)=\frac{1}{2} \varphi^{(0)}+\frac{1}{2} \varphi^{(1)}, \\
\varphi^{(0)}(132) & =-\varphi^{(0)}(312)=\frac{1}{2} \varphi^{(0)}-\frac{1}{2} \varphi^{(1)} \\
\varphi^{(1)}(123)=\varphi^{(1)}(213)=\varphi^{(1)}, \quad(321) & =\varphi^{(1)}(231)=\frac{3}{2} \varphi^{(0)}-\frac{1}{2} \varphi^{(1)}, \\
\varphi^{(1)}(132) & =\varphi^{(1)}(312)=-\frac{3}{2} \varphi^{(0)}-\frac{1}{2} \varphi^{(1)} .
\end{aligned}
$$

We take as $\psi(123)$ in the formulas of sect. 5.1 the spin wave function $\varphi(123)$ of the general form:

$$
\varphi(123)=a \varphi^{(1)}(123)+b \varphi^{(0)}(123) .
$$

Substituting (44) in eqs. (28), (29) and using (43) we reproduce the well known result:

$$
\varphi_{S}=0, \quad \varphi_{A}=0 .
$$

Substituting (44) in eqs. (30), for the functions with mixed symmetry we find:

$$
\left\{\begin{array}{l}
\varphi_{1} \propto 3 a\left(-\varphi^{(0)}+\varphi^{(1)}\right), \\
\varphi_{2} \propto 3 a\left(\varphi^{(0)}+\varphi^{(1)}\right) .
\end{array}\right.
$$

The second mixed symmetry doublet $\varphi_{1}^{\prime}, \varphi_{2}^{\prime}$, eq. (34), coincides (up to a factor) with $\varphi_{1}, \varphi_{2}$.

Denote:

$$
\left\{\begin{array}{l}
\varphi_{1}=-\varphi^{(0)}+\varphi^{(1)}=-1+\vec{\sigma}_{12} \cdot \vec{\sigma}_{3 N} \\
\varphi_{2}=\varphi^{(0)}+\varphi^{(1)}=1+\vec{\sigma}_{12} \cdot \vec{\sigma}_{3 N} .
\end{array}\right.
$$

These functions are forming the mixed symmetry doublet of the type (30). The normalization factor will be determined for full wave function.

By this way, after permutations we have reduced the spin wave function to the form (45) with the "canonical" order of spinors $(12)(3 N)$, when 1 is coupled with 2 and 3 with $N$. In principle, this is not obligatory, but is very convenient. We will see below in sect. 9 that in the form factor calculations this results in the product of two traces, each of them containing approximately half of the Dirac matrices involved, instead of the trace of full product. This simplifies calculations considerably. 
Similarly to (45) we introduce the isospin functions:

$$
\left\{\begin{array}{l}
\chi_{1}=-\chi^{(0)}+\chi^{(1)}=-1+\vec{\tau}_{12} \cdot \vec{\tau}_{3 N} \\
\chi_{2}=\chi^{(0)}+\chi^{(1)}=1+\vec{\tau}_{12} \cdot \vec{\tau}_{3 N}
\end{array}\right.
$$

where

$$
1=\left(\xi_{1}^{\dagger} \tau_{y} \xi_{2}^{*}\right)\left(\xi_{3}^{\dagger} \xi_{N}\right) \equiv \chi^{(0)}, \quad \vec{\tau}_{12} \cdot \vec{\tau}_{3 N}=\left(\xi_{1}^{\dagger} \vec{\tau} \tau_{y} \xi_{2}^{*}\right) \cdot\left(\xi_{3}^{\dagger} \vec{\tau} \xi_{N}\right) \equiv \chi^{(1)}
$$

$\xi$ 's are the isospin spinors. These functions satisfy the symmetry properties (43). Like in the case of spin, $\chi_{S}=\chi_{A}=0$.

From the above mixed symmetry spin and isospin doublets we can construct the spin-isospin functions with given symmetry.

Substituting (45) and (46) into the equation (36), we get the symmetric spin-isospin function. Multiplying it by the symmetric $\psi_{S}$, we find the total symmetric nucleon wave function:

$$
\Psi_{S}=\frac{1}{\sqrt{72}} \psi_{S}\left[3+\left(\vec{\sigma}_{12} \cdot \vec{\sigma}_{3 N}\right)\left(\vec{\tau}_{12} \cdot \vec{\tau}_{3 N}\right)\right]
$$

It is given in many papers (compare, for example, with [9]). The invariant symmetric function $\psi_{S}$ depends on the relative momenta and on $\vec{n}: \psi_{S}=\psi_{S}\left(\vec{q}_{1}, \overrightarrow{q_{2}}, \overrightarrow{q_{3}}, \vec{n}\right)$. The normalization factor will be explained in the next section.

Substituting (45) and (46) into the equation (37), we get for the antisymmetric spin-isospin function. Multiplying it by antisymmetric $\psi_{A}$, we find:

$$
\Psi_{S}=\frac{1}{\sqrt{24}} \psi_{A}\left[\vec{\sigma}_{12} \cdot \vec{\sigma}_{3 N}-\vec{\tau}_{12} \cdot \vec{\tau}_{3 N}\right]
$$

Substituting (45) and (46) into (38), we obtain the mixed symmetry functions:

$$
\left\{\begin{array}{l}
\phi_{1}=3-\vec{\tau}_{12} \cdot \vec{\tau}_{3 N}-\vec{\sigma}_{12} \cdot \vec{\sigma}_{3 N}-\left(\vec{\sigma}_{12} \cdot \vec{\sigma}_{3 N}\right)\left(\vec{\tau}_{12} \cdot \vec{\tau}_{3 N}\right) \\
\phi_{2}=3+\vec{\tau}_{12} \cdot \vec{\tau}_{3 N}+\vec{\sigma}_{12} \cdot \vec{\sigma}_{3 N}-\left(\vec{\sigma}_{12} \cdot \vec{\sigma}_{3 N}\right)\left(\vec{\tau}_{12} \cdot \vec{\tau}_{3 N}\right)
\end{array}\right.
$$

Substituting eqs. (50) into (41) we get:

$$
\begin{aligned}
\Psi_{S} & =\frac{1}{\sqrt{288}} \psi_{1}\left[3-3 \vec{\tau}_{12} \cdot \vec{\tau}_{3 N}-3 \vec{\sigma}_{12} \cdot \vec{\sigma}_{3 N}-\left(\vec{\sigma}_{12} \cdot \vec{\sigma}_{3 N}\right)\left(\vec{\tau}_{12} \cdot \vec{\tau}_{3 N}\right)\right] \\
& +\frac{1}{\sqrt{288}} \psi_{2}\left[3+3 \vec{\tau}_{12} \cdot \vec{\tau}_{3 N}+3 \vec{\sigma}_{12} \cdot \vec{\sigma}_{3 N}-\left(\vec{\sigma}_{12} \cdot \vec{\sigma}_{3 N}\right)\left(\vec{\tau}_{12} \cdot \vec{\tau}_{3 N}\right)\right]
\end{aligned}
$$

\subsection{Normalization}

To calculate the normalization, we use the equalities

$$
\begin{aligned}
\varphi^{\dagger(0)} \varphi^{(0)} & =\frac{1}{2} \sum_{\sigma, \sigma_{1}, \sigma_{2}, \sigma_{3}}\left[\left(w_{2}^{\sigma_{2}} \sigma_{y} w_{1}^{\sigma_{1}}\right)\left(w_{1}^{\dagger \sigma_{1}} \sigma_{y} w_{2}^{* \sigma_{2}}\right)\right]\left[\left(w_{N}^{\dagger \sigma} w_{3}^{\sigma_{3}}\right)\left(w_{3}^{\dagger \sigma_{3}} w_{N}^{\sigma}\right)\right] \\
& =\frac{1}{2} \operatorname{Tr}\left[\sigma_{y}^{2}\right] \operatorname{Tr}[1]=2
\end{aligned}
$$




$$
\begin{aligned}
\varphi^{\dagger(1)} \varphi^{(1)} & =\frac{1}{2} \sum_{\sigma, \sigma_{1}, \sigma_{2}, \sigma_{3}}\left[\left(w_{2}^{\sigma_{2}} \sigma_{i} \sigma_{y} w_{1}^{\sigma_{1}}\right)\left(w_{1}^{\dagger \sigma_{1}} \sigma_{y} \sigma_{j} w_{2}^{* \sigma_{2}}\right)\right]\left[\left(w_{N}^{\dagger \sigma} \sigma_{i} w_{3}^{\sigma_{3}}\right)\left(w_{3}^{\dagger \sigma_{3}} \sigma_{j} w_{N}^{\sigma}\right)\right] \\
& =\frac{1}{2} \operatorname{Tr}\left[\sigma_{j} \sigma_{i}\right] \operatorname{Tr}\left[\sigma_{i} \sigma_{j}\right]=6, \\
\varphi^{\dagger(1)} \varphi^{(0)} & =\varphi^{\dagger(0)} \varphi^{(1)}=0,
\end{aligned}
$$

and similarly for the isospin functions $\chi^{(0)}, \chi^{(1)}$. The factor $\frac{1}{2}$ in these formulas appears due to averaging over the nucleon spin projection $\sigma$. Then for the function (48) we find:

$$
\overline{\Psi_{S}^{\dagger} \Psi_{S}}=\frac{1}{72}\left(3^{2} \times 2 \times 2+6 \times 6\right) \psi_{S}^{2}=\psi_{S}^{2} .
$$

The factor $2 \times 2$ in the first item comes from the product of the spin and isospin functions $\varphi^{(0)}, \chi^{(0)}$, the factor $6 \times 6$ in the second item comes from the product of $\varphi^{(1)}, \chi^{(1)}$.

For the function (49) with antisymmetric $\psi_{A}$ we get:

$$
\overline{\Psi_{S}^{\dagger} \Psi_{S}}=\frac{1}{24}(6 \times 2+2 \times 6) \psi_{A}^{2}=\psi_{A}^{2},
$$

and for the mixed symmetry $\psi_{1,2}$, eq. (51):

$$
\overline{\Psi_{S}^{\dagger} \Psi_{S}}=\left(\psi_{1}^{2}+\psi_{2}^{2}-\psi_{1} \psi_{2}\right)
$$

Provided the function $\psi_{S}$ dominates, it is normalized as

$$
\int \psi_{S}^{2} D=1
$$

If $\psi_{S}$ does not dominate, the integral (56) is not equal to 1 , but gives the contribution of $\psi_{S}$ into full normalization integral. The contributions of symmetric and mixed symmetry states are given by the similar integrals from $\psi_{A}^{2}$ and $\left(\psi_{1}^{2}+\psi_{2}^{2}-\psi_{1} \psi_{2}\right)$.

In eq. (56) we introduced for shortness the notation for the integration volume [18, 23]:

$$
\begin{aligned}
D & \equiv(2 \pi)^{3} \delta^{(3)}\left(\sum_{i=1}^{3} \vec{q}_{i}\right) 2\left(\sum_{i=1}^{3} \varepsilon_{q_{i}}\right) \prod_{i=1}^{3} \frac{d^{3} q_{i}}{(2 \pi)^{3} 2 \varepsilon_{q_{i}}} . \\
& =(2 \pi)^{3} \delta^{(2)}\left(\sum_{i=1}^{3} \vec{R}_{i \perp}\right) \delta\left(\sum_{i=1}^{3} x_{i}-1\right) 2 \prod_{i=1}^{3} \frac{d^{2} R_{i \perp}}{(2 \pi)^{3}} \frac{d x_{i}}{2 x_{i}}
\end{aligned}
$$

The last line of (57) is written in the variables which are similar to the well known infinite momentum frame variables. Namely, $x_{i}$ is defined as $x_{i}=\omega \cdot k_{i} / \omega \cdot p$. Then we introduce the four-vectors: $R_{i}=k_{i}-x_{i} p$. They satisfy the condition $R_{i} \cdot \omega=0$ and can be represented as $R_{i}=\left(R_{i 0}, \vec{R}_{i \perp}, \vec{R}_{i \|}\right)$, where $\vec{R}_{i \|}$ is parallel to $\vec{\omega}$ and $\vec{R}_{i \perp} \cdot \vec{\omega}=0$. So, $\vec{R}_{i \perp}$ are the two-dimensional vectors such that $\vec{R}_{i \perp}^{2}=-R_{i}^{2}$. We thus have the following relations:

$$
\vec{R}_{1 \perp}+\vec{R}_{2 \perp}+\vec{R}_{3 \perp}=0, \quad x_{1}+x_{2}+x_{3}=1,
$$


like for the infinite momentum frame variables.

For convenience, we can express $\psi_{S}$ in (48) through another scalar function $\varphi_{0}$ :

$$
\psi_{S}=\frac{2}{\sqrt{3}} N m \varphi_{0},
$$

with the normalization

$$
\int \varphi_{0}^{2}(2 \pi)^{3} \delta^{(3)}\left(\vec{q}_{1}+\vec{q}_{2}+\vec{q}_{3}\right) \prod_{i=1}^{3} \frac{d^{3} q_{i}}{(2 \pi)^{3}}=1 .
$$

The dimensionless normalization constant $N$ in (58) is found from the condition obtained by substituting (58) into (56):

$$
\frac{4}{3} N^{2} m^{2} \int \varphi_{0}^{2} D=1 .
$$

In the nonrelativistic limit the factor $D$ turns into the integration volume of eq. (59) multiplied by $3 /\left(4 m^{2}\right)$. Therefore $N$ tends to 1 .

For $\varphi_{0}$ we can take, for example, the harmonic oscillator model:

$$
\varphi_{0}=\frac{2^{3} \pi^{3 / 2} 3^{3 / 4}}{\alpha^{3}} \exp \left(-\frac{{\overrightarrow{q_{1}}}^{2}+{\overrightarrow{q_{2}}}^{2}+\vec{q}_{3}^{2}}{2 \alpha^{2}}\right) .
$$

\subsection{Four-dimensional representation}

In the section 3 the spin operators $\vec{\sigma}_{12}, \vec{\sigma}_{3 N}$ were constructed through the spinors $u_{\mathcal{P}}$ in the special representation (12). In this section we represent the structures given above through the usual spinors $u$. This allows to use in calculations the standard trace techniques. Similar representation for the components (25) on the energy shell was found in [7].

Since the spinors in two representations are related with each other by eq. (12), the standard representation is obtained by replacing $u_{\mathcal{P}}$ by $u$. They coincide with each other in the reference frame where $\overrightarrow{\mathcal{P}}$. So, from (15) we find that:

$$
\vec{\sigma}_{12} \cdot \vec{\sigma}_{3 N} \rightarrow-c_{1} c_{2} c_{3} c_{N}\left[\bar{u}\left(k_{1}\right) \Pi_{+} \gamma^{\mu} \Pi_{-} U_{c} \bar{u}\left(k_{2}\right)\right]\left[\bar{u}\left(k_{3}\right) \Pi_{+} \gamma_{\mu} \gamma_{5} \Pi_{+} u(p)\right] .
$$

The scalar product is defined as $a \cdot b=a_{\mu} b^{\mu}=a_{0} b^{0}-\vec{a} \cdot \vec{b}$. At $\overrightarrow{\mathcal{P}}=0$ we have $\Pi_{+} \gamma^{0} \Pi_{-}=$ $\Pi_{+} \gamma^{0} \gamma_{5} \Pi_{+}=0$, and we see that r.h.-side of (62) indeed coincides with $\vec{\sigma}_{12} \cdot \vec{\sigma}_{3 N}$.

Similarly we obtain for the unit operators:

$$
1_{12}=\left(w_{1}^{\dagger} \sigma_{y} w_{2}^{*}\right) \rightarrow c_{1} c_{2} \bar{u}\left(k_{1}\right) \Pi_{+} \gamma_{5} \Pi_{-} U_{c} \bar{u}\left(k_{2}\right), \quad 1_{3 N}=\left(w_{3}^{\dagger} w_{N}\right) \rightarrow c_{3} c_{N} \bar{u}\left(k_{3}\right) \Pi_{+} \Pi_{+} u(p) .
$$

In eqs. (63) we can substitute $\Pi_{+} \gamma_{5} \Pi_{-}=\Pi_{+} \gamma_{5}, \Pi_{+} \Pi_{+}=\Pi_{+}$.

Using the above formulas we represent in the four-dimensional form the functions with the spin structures independent of momenta. The symmetric structure (48) is represented as:

$$
\begin{aligned}
\Psi_{S}= & \frac{\psi_{S}}{\sqrt{72}} c_{1} c_{2} c_{3} c_{N}\left\{3\left[\bar{u}\left(k_{1}\right) \Pi_{+} \gamma_{5} U_{c} \bar{u}\left(k_{2}\right)\right]\left[\bar{u}\left(k_{3}\right) \Pi_{+} u(p)\right]\right. \\
& \left.-\left[\bar{u}\left(k_{1}\right) \Pi_{+} \gamma^{\mu} \Pi_{-} U_{c} \bar{u}\left(k_{2}\right)\right]\left[\bar{u}\left(k_{3}\right) \Pi_{+} \gamma_{\mu} \gamma_{5} \Pi_{+} u(p)\right]\left(\vec{\tau}_{12} \cdot \vec{\tau}_{3 N}\right)\right\} \\
\equiv & \Psi_{0}+\Psi_{1}
\end{aligned}
$$


where $c_{i}$ are defined by (16). For applications given below we denote two items in (64) as $\Psi_{0}$ and $\Psi_{1}$.

The antisymmetric structure (49) reads:

$$
\begin{aligned}
\Psi_{S}= & -\frac{\psi_{A}}{\sqrt{24}} c_{1} c_{2} c_{3} c_{N}\left\{\left[\bar{u}\left(k_{1}\right) \Pi_{+} \gamma^{\mu} \Pi_{-} U_{c} \bar{u}\left(k_{2}\right)\right]\left[\bar{u}\left(k_{3}\right) \Pi_{+} \gamma_{\mu} \gamma_{5} \Pi_{+} u(p)\right]\right. \\
& \left.+\left[\bar{u}\left(k_{1}\right) \Pi_{+} \gamma_{5} U_{c} \bar{u}\left(k_{2}\right)\right]\left[\bar{u}\left(k_{3}\right) \Pi_{+} u(p)\right]\left(\vec{\tau}_{12} \cdot \vec{\tau}_{3 N}\right)\right\}
\end{aligned}
$$

The mixed symmetry function (51) is given by:

$$
\begin{array}{r}
\Psi_{S}=\frac{\psi_{1}}{\sqrt{288}} c_{1} c_{2} c_{3} c_{N}\left\{3\left[\bar{u}\left(k_{1}\right) \Pi_{+} \gamma_{5} U_{c} \bar{u}\left(k_{2}\right)\right]\left[\bar{u}\left(k_{3}\right) \Pi_{+} u(p)\right]\left(1-\vec{\tau}_{12} \cdot \vec{\tau}_{3 N}\right)\right. \\
\left.\quad+\left[\bar{u}\left(k_{1}\right) \Pi_{+} \gamma^{\mu} \Pi_{-} U_{c} \bar{u}\left(k_{2}\right)\right]\left[\bar{u}\left(k_{3}\right) \pi_{+} \gamma_{\mu} \gamma_{5} \pi_{+} u(p)\right]\left(3+\vec{\tau}_{12} \cdot \vec{\tau}_{3 N}\right)\right\} \\
+\frac{\psi_{2}}{\sqrt{288}} c_{1} c_{2} c_{3} c_{N}\left\{3\left[\bar{u}\left(k_{1}\right) \Pi_{+} \gamma_{5} U_{c} \bar{u}\left(k_{2}\right)\right]\left[\bar{u}\left(k_{3}\right) \Pi_{+} u(p)\right]\left(1+\vec{\tau}_{12} \cdot \vec{\tau}_{3 N}\right)\right. \\
\left.-\left[\bar{u}\left(k_{1}\right) \Pi_{+} \gamma^{\mu} \Pi_{-} U_{c} \bar{u}\left(k_{2}\right)\right]\left[\bar{u}\left(k_{3}\right) \Pi_{+} \gamma_{\mu} \gamma_{5} \Pi_{+} u(p)\right]\left(3-\vec{\tau}_{12} \cdot \vec{\tau}_{3 N}\right)\right\} .
\end{array}
$$

The above expressions can be represented in the form of decomposition in terms of the spin structures (25-26). They become more lengthy, and we will not give them here. The expressions (64 66) are enough for calculations. Note, however, that the "old" structures (25) are not enough for this decomposition. For example, the decomposition of the symmetric function (64) contains the terms from (26), constructed by means of the pseudoscalar $C_{p s}$, eq. (27).

We give also the four-dimensional form of the normalization in the example of symmetric wave function. Its contribution to the normalization integral has the form:

$$
\begin{aligned}
& \int \overline{\Psi_{S}^{\dagger} \Psi_{S}} D, \quad \text { where } \overline{\Psi_{S}^{\dagger} \Psi_{S}}=\frac{1}{2 \cdot 72} \psi_{S}^{2} c_{1}^{2} c_{2}^{2} c_{3}^{2} c_{N}^{2} \\
\times & \left\{3^{2} 2 \operatorname{Tr}\left[\Pi_{-} \gamma_{5} \Pi_{+}\left(\hat{k}_{1}+m\right) \Pi_{+} \gamma_{5} \Pi_{-}\left(-\hat{k}_{2}+m\right)\right] \operatorname{Tr}\left[\Pi_{+}\left(\hat{k}_{3}+m\right) \Pi_{+}(\hat{p}+M)\right]\right. \\
+ & 6 \operatorname{Tr}\left[\Pi_{-} \gamma^{\nu} \Pi_{+}\left(\hat{k}_{1}+m\right) \Pi_{+} \gamma^{\mu} \Pi_{-}\left(-\hat{k}_{2}+m\right)\right] \\
\times & \left.\operatorname{Tr}\left[\Pi_{+} \gamma_{5} \gamma_{\nu} \Pi_{+}\left(\hat{k}_{3}+m\right) \Pi_{+} \gamma_{\mu} \gamma_{5} \Pi_{+}(\hat{p}+M)\right]\right\} .
\end{aligned}
$$

This formula can be simplified, using the equalities

$$
\Pi_{+}\left(\hat{k_{1}}+m\right) \Pi_{+}=\Pi_{+} / c_{1}^{2}, \quad \Pi_{-}\left(-\hat{k_{2}}+m\right) \Pi_{-}=\Pi_{-} / c_{2}^{2}, \quad \text { etc. }
$$

By this way we get:

$$
\begin{aligned}
\overline{\Psi_{S}^{\dagger} \Psi_{S}} & =\psi_{S}^{2} \frac{1}{2 \cdot 72}\left\{3^{2} 2 \operatorname{Tr}\left[\Pi_{+}\right] \operatorname{Tr}\left[\Pi_{+}\right]+6 \operatorname{Tr}\left[\Pi_{-} \gamma^{\nu} \Pi_{+} \gamma^{\mu}\right] \operatorname{Tr}\left[\Pi_{-} \gamma_{\nu} \Pi_{+} \gamma_{\mu}\right]\right\} \\
& =\psi_{S}^{2} \frac{1}{2 \cdot 72}\left\{3^{2} \times 2 \times 2 \times 2+6 \times 12\right\}=\psi_{S}^{2}
\end{aligned}
$$

that coincides with the normalization (53). 


\section{The components depending on momenta}

\subsection{Symmetric tensors}

Let us construct now the depending on momenta spin components with the given symmetry. We start with the tensors (20), symmetric in the indices $i j$. However, they have no any definite symmetry relative to the permutations of the particles. We can construct the symmetric relative to the permutations function:

$$
f_{S}=T_{11}+T_{22}+T_{33}=2 T_{11}+2 T_{22}+T_{12}
$$

and two doublets with mixed symmetry:

$$
\left\{\begin{array} { l } 
{ f _ { 1 } = T _ { 1 1 } - T _ { 3 3 } = - T _ { 2 2 } - T _ { 1 2 } } \\
{ f _ { 2 } = T _ { 2 2 } - T _ { 3 3 } = - T _ { 1 1 } - T _ { 1 2 } }
\end{array} \quad ( a ) \quad \left\{\begin{array}{l}
f_{1}^{\prime}=T_{1 n}-T_{3 n}=2 T_{1 n}+T_{2 n} \\
f_{2}^{\prime}=T_{2 n}-T_{3 n}=2 T_{2 n}+T_{1 n}
\end{array} \quad(b)\right.\right.
$$

For shortness we omit the indices $i j$. One can easily check that the functions (70) indeed satisfy the permutation properties (32). It is impossible to construct any antisymmetric structure.

We represent also the spin tensor $\sigma_{12}^{i} \sigma_{3 N}^{j}$ in the form of symmetric in the indices $i j$ traceless tensor:

$$
S^{i j}=\sigma_{12}^{i} \sigma_{3 N}^{j}+\sigma_{12}^{j} \sigma_{3 N}^{i}-\frac{2}{3} \vec{\sigma}_{12} \cdot \vec{\sigma}_{3 N} \delta^{i j}
$$

Using the Fierz identities given in appendix D we find that $S^{i j}$ is fully symmetric also relative to permutation of any pair of the particles $1,2,3$. This reflects the fact that there is no any other traceless symmetric tensor made of $\vec{\sigma}_{12}, \vec{\sigma}_{3 N}$.

The depending on momenta spin structures $\varphi_{S}, \varphi_{1,2}, \varphi_{1,2}^{\prime}$ are obtained by the contracting of $f_{S}$, eq. (69), and $f_{1,2}, f_{1,2}^{\prime}$, eq. (70), with the spin operator $S$ :

$$
\left(\varphi_{S}, \varphi_{1,2}, \varphi_{1,2}^{\prime}\right)=\left(f_{S}, f_{1,2}, f_{1,2}^{\prime}\right) \times S
$$

This contraction does not change the symmetry of the functions $f$ 's. Therefore the symmetry of $\varphi$ 's coincides with the symmetry of $f$ 's.

By the standard formulas of the section 5.2 we construct from $\varphi$ and from the isospin functions $\chi$ the spin-isospin functions $\phi$ of all symmetries: symmetric, antisymmetric and mixed symmetry. Then from these functions $\phi$ and from the invariant momentum functions $\psi$ of different symmetries, by the formulas (39-41), we construct the total symmetric wave function of the nucleon.

For example, contracting $S$ with the functions $f_{1,2}$, eq. (70), we get the mixed symmetry spin functions:

$$
\left\{\begin{array}{l}
\varphi_{1}=T_{11}^{i j} S_{i j}-T_{33}^{i j} S_{i j} \\
\varphi_{2}=T_{22}^{i j} S_{i j}-T_{33}^{i j} S_{i j}
\end{array}\right.
$$

Then we substitute these functions $\varphi_{1,2}$ and the mixed symmetry isospin functions $\chi_{1,2}$ (46) into (36) and get the fully symmetric spin-isospin function $\phi_{S}$. Multiplied by $\psi_{S}$, eq. 
(39), it gives the total fully symmetric nucleon wave function:

$$
\begin{aligned}
\Psi_{S}= & \psi_{S}\left\{\left[\left(\vec{q}_{1} \cdot \vec{\sigma}_{12}\right)\left(\vec{q}_{1} \cdot \vec{\sigma}_{3 N}\right)-\frac{1}{3} \vec{q}_{1}^{2}\left(\vec{\sigma}_{12} \cdot \vec{\sigma}_{3 N}\right)\right]\left(\vec{\tau}_{12} \cdot \vec{\tau}_{3 N}-3\right)\right. \\
+ & {\left[\left(\vec{q}_{2} \cdot \vec{\sigma}_{12}\right)\left(\vec{q}_{2} \cdot \vec{\sigma}_{3 N}\right)-\frac{1}{3} \vec{q}_{2}^{2}\left(\vec{\sigma}_{12} \cdot \vec{\sigma}_{3 N}\right)\right]\left(\vec{\tau}_{12} \cdot \vec{\tau}_{3 N}+3\right) } \\
- & \left.2\left[\left(\vec{q}_{3} \cdot \vec{\sigma}_{12}\right)\left(\vec{q}_{3} \cdot \vec{\sigma}_{3 N}\right)-\frac{1}{3} \vec{q}_{3}^{2}\left(\vec{\sigma}_{12} \cdot \vec{\sigma}_{3 N}\right)\right] \vec{\tau}_{12} \cdot \vec{\tau}_{3 N}\right\}
\end{aligned}
$$

Similarly we find all other functions forming the basis of the nucleon wave function. The full set of these functions is given in appendix $\mathrm{E}$.

\subsection{Antisymmetric tensors}

Now consider the tensors (23), antisymmetric in the indices $i j$. Introduce the spin operators antisymmetric in $i j$ :

$$
\Gamma^{i j}(123)=\sigma_{12}^{i} \sigma_{3 N}^{j}-\sigma_{12}^{j} \sigma_{3 N}^{i}, \Gamma_{+}^{i j}(123)=-i \epsilon_{i j k}\left(\sigma_{12}^{k}+\sigma_{3 N}^{k}\right), \Gamma_{-}^{i j}(123)=-i \epsilon_{i j k}\left(\sigma_{12}^{k}-\sigma_{3 N}^{k}\right) .
$$

Using the Fierz identities, appendix D, one can establish the following properties relative to permutations of the particles:

$$
\begin{array}{lll}
\Gamma(231)=\Gamma(321)=\Gamma_{-}(123), & \Gamma(312)=\Gamma(132)=\Gamma_{+}(123), & \Gamma(213)=\Gamma(123), \\
\Gamma_{+}(213)=\Gamma_{+}(312)=\Gamma_{-}(123), & \Gamma_{+}(231)=\Gamma_{+}(132)=\Gamma(123), & \Gamma_{+}(321)=\Gamma_{+}(123), \\
\Gamma_{-}(213)=\Gamma_{-}(231)=\Gamma_{+}(123), & \Gamma_{-}(321)=\Gamma_{-}(312)=\Gamma(123), & \Gamma_{-}(132)=\Gamma_{-}(123) .
\end{array}
$$

From three operators (74) we construct another three operators forming the symmetric representation of the permutation group $\Sigma_{S}$ and the mixed symmetry one $\Sigma_{1,2}$ :

$$
\begin{gathered}
\Sigma_{S}=\Gamma+\Gamma_{+}+\Gamma_{-} \\
\left\{\begin{array}{l}
\Sigma_{1}=\Gamma-\Gamma_{-} \\
\Sigma_{2}=\Gamma-\Gamma_{+}
\end{array}\right.
\end{gathered}
$$

From the tensors (23) we construct antisymmetric and the mixed symmetry functions:

$$
\begin{gathered}
f_{A}=A_{12} \\
\left\{\begin{array}{c}
f_{1}=A_{1 n}-A_{3 n}=2 A_{1 n}+A_{2 n} \\
f_{2}=A_{2 n}-A_{3 n}=2 A_{2 n}+A_{1 n}
\end{array}\right.
\end{gathered}
$$

It is impossible to construct any symmetric function.

Using the formulas from sect. 5.2 we find the spin wave functions $\varphi$ with given symmetry. Namely:

$$
\begin{gathered}
\varphi_{S}=2\left(f_{1} \Sigma_{1}+f_{2} \Sigma_{2}\right)-f_{1} \Sigma_{2}-f_{2} \Sigma_{1}, \\
\varphi_{A}=f_{2} \Sigma_{1}-f_{1} \Sigma_{2} \\
\varphi_{A}=f_{A} \Sigma_{S}
\end{gathered}
$$


and

$$
\left\{\begin{array} { l } 
{ \varphi _ { 1 } = \Sigma _ { S } f _ { 1 } } \\
{ \varphi _ { 2 } = \Sigma _ { S } f _ { 2 } }
\end{array} ( a ) \quad \left\{\begin{array} { l } 
{ \varphi _ { 1 } = f _ { A } ( \Sigma _ { 1 } - 2 \Sigma _ { 2 } ) } \\
{ \varphi _ { 2 } = f _ { A } ( 2 \Sigma _ { 1 } - \Sigma _ { 2 } ) }
\end{array} ( b ) \quad \left\{\begin{array}{l}
\varphi_{1}=\left(f_{1}-f_{2}\right) \Sigma_{1}-f_{1} \Sigma_{2} \\
\varphi_{2}=-f_{2} \Sigma_{1}+\left(f_{2}-f_{1}\right) \Sigma_{2}
\end{array}(\text { c) }\right.\right.\right.
$$

In the above formulas we imply the summation over the indices $i j$.

Then again by means of eqs. (36-38) we construct from these $\varphi$ 's and the isospin functions $\chi$ 's the spin-isospin functions $\phi$ and then, by eqs. (39-41), from $\phi$ 's and the momentum functions $\psi$ 's we obtain the total nucleon wave function $\Psi_{S}$. As an example, we give here a wave function with the symmetric spin-isospin and the scalar parts:

$$
\Psi_{S}=\psi_{S}\left\{\left(\vec{q}_{1} \cdot \vec{\sigma}_{12}\right)\left(\vec{q}_{2} \cdot \vec{\sigma}_{3 N}\right)-\left(\vec{q}_{2} \cdot \vec{\sigma}_{12}\right)\left(\vec{q}_{1} \cdot \vec{\sigma}_{3 N}\right)+i \vec{\sigma}_{12} \cdot\left[\vec{q}_{1} \times \vec{q}_{2}\right]-i \vec{\sigma}_{3 N} \cdot\left[\vec{q}_{1} \times \vec{q}_{2}\right] \vec{\tau}_{12} \cdot \vec{\tau}_{3 N}\right\}
$$

The full set of the corresponding wave functions is given in appendix $\mathrm{F}$.

\subsection{Four-dimensional representation}

The operators $\vec{\sigma}_{12} \cdot \vec{\sigma}_{3 N}$ and 1 are already represented through the Dirac matrices and the bi-spinors by the formulas (62) and (63). In this section we give the four-dimensional form of other structures.

Any scalar products of $\vec{\sigma}_{1,2}$ with the vectors $\vec{q}_{1,2,3}, \vec{n}$ can be represented similarly to (62). For example:

$$
\begin{aligned}
& \left(\vec{q}_{2} \cdot \vec{\sigma}_{12}\right) \rightarrow c_{1} c_{2} \bar{u}\left(k_{1}\right) \Pi_{+} \hat{k}_{2} \Pi_{-} U_{c} \bar{u}\left(k_{2}\right), \quad\left(\vec{q}_{1} \cdot \vec{\sigma}_{3 N}\right) \rightarrow c_{3} c_{N} \bar{u}\left(k_{3}\right) \Pi_{+} \hat{k}_{1} \gamma_{5} \Pi_{+} u(p) \\
& \left(\vec{n} \cdot \vec{\sigma}_{12}\right) \rightarrow c_{1} c_{2} \frac{\mathcal{M}}{\omega \cdot p} \bar{u}\left(k_{1}\right) \Pi_{+} \hat{\omega} \Pi_{-} U_{c} \bar{u}\left(k_{2}\right), \quad\left(\vec{n} \cdot \vec{\sigma}_{3 N}\right) \rightarrow c_{3} c_{N} \frac{\mathcal{M}}{\omega \cdot p} \bar{u}\left(k_{3}\right) \Pi_{+} \hat{\omega} \gamma_{5} \Pi_{+} u(p),
\end{aligned}
$$

etc. At $\overrightarrow{\mathcal{P}}=0, \mathcal{P}_{0}=\mathcal{M}$ the factor $\mathcal{M} / \omega \cdot p=\mathcal{M} / \omega \cdot \mathcal{P}$ turns into $1 / \omega_{0}$, and $\vec{\omega} / \omega_{0}$ gives $\vec{n}$.

One can similarly represent the expressions containing the vector products. For example:

$$
\begin{aligned}
\vec{\sigma}_{12} \cdot\left[\vec{q}_{1} \times \vec{q}_{2}\right] & \rightarrow-\epsilon^{\mu \nu \rho \gamma} c_{1} c_{2}\left[\bar{u}\left(k_{1}\right) \Pi_{+} \gamma_{\mu} \Pi_{-} U_{c} \bar{u}\left(k_{2}\right)\right] k_{1 \nu} k_{2 \rho} \mathcal{P}_{\gamma} / \mathcal{M}, \\
\vec{\sigma}_{3 N} \cdot\left[\vec{q}_{1} \times \vec{q}_{2}\right] & \rightarrow-\epsilon^{\mu \nu \rho \gamma} c_{3} c_{N}\left[\bar{u}\left(k_{3}\right) \Pi_{+} \gamma_{\mu} \gamma_{5} \Pi_{+} u(p)\right] k_{1 \nu} k_{2 \rho} \mathcal{P}_{\gamma} / \mathcal{M}, \\
\vec{\sigma}_{12} \cdot\left[\vec{q}_{1} \times \vec{n}\right] & \rightarrow-\epsilon^{\mu \nu \rho \gamma} c_{1} c_{2}\left[\bar{u}\left(k_{1}\right) \Pi_{+} \gamma_{\mu} \Pi_{-} U_{c} \bar{u}\left(k_{2}\right)\right] k_{1 \nu} \omega_{\rho} \mathcal{P}_{\gamma} / \omega \cdot p, \\
\vec{\sigma}_{3 N} \cdot\left[\vec{q}_{1} \times \vec{n}\right] & \rightarrow-\epsilon^{\mu \nu \rho \gamma} c_{3} c_{N}\left[\bar{u}\left(k_{3}\right) \Pi_{+} \gamma_{\mu} \gamma_{5} \Pi_{+} u(p)\right] k_{1 \nu} \omega_{\rho} \mathcal{P}_{\gamma} / \omega \cdot p,
\end{aligned}
$$

etc. By these substitutions the nucleon wave function can be easily represented in the four-dimensional form. We do not give here the explicit formulas. 


\section{Matrix elements of the current operator}

\subsection{The quark current}

The quark electromagnetic current has the form:

$$
J_{\rho}=\sum_{i=1,2,3} \bar{u}_{i}^{\prime} \gamma_{\rho} u_{i}\left[\xi_{i}^{\dagger} \tau_{i}^{\prime} \frac{1}{2}\left(\frac{1}{3}+\tau_{z}^{(i)}\right) \xi_{i}^{\tau_{i}}\right] \Rightarrow 3 \bar{u}^{\prime}\left(k_{3}\right) \gamma_{\rho} u\left(k_{3}\right)\left[\xi_{3}^{\dagger \tau_{3}^{\prime}} \frac{1}{2}\left(\frac{1}{3}+\tau_{z}^{(3)}\right) \xi_{3}^{\tau_{3}}\right]
$$

$\tau_{z}^{(i)}$ is the Pauli matrix acting on the spinor of the $i$-th quark. The replacement by the only item has been made due to symmetry of the wave function to be used for calculating the matrix elements. In (86) the factor

$$
\frac{1}{2}\left(\frac{1}{3}+\tau_{z}\right)=\left(\begin{array}{cc}
2 / 3 & 0 \\
0 & -1 / 3
\end{array}\right)
$$

reproduces the charges of $u$ and $d$ quarks.

The quark axial current has the form:

$$
J_{\rho}=\sum_{i=1,2,3} \bar{u}_{i}^{\prime} \gamma_{\rho} \gamma_{5} u_{i}\left[\xi_{i}^{\dagger \tau_{i}^{\prime}} \vec{\tau}^{(i)} \xi_{i}^{\tau_{i}}\right] \Rightarrow 3 \bar{u}^{\prime}\left(k_{3}\right) \gamma_{\rho} \gamma_{5} u\left(k_{3}\right)\left[\xi_{3}^{\dagger \tau_{3}^{\prime}} \vec{\tau}^{(3)} \xi_{3}^{\tau_{3}}\right]
$$

At first, we calculate the isospin matrix elements relative to the isospin wave functions $\chi^{(0)}, \chi^{(1)}$ given by (47). Let $I$ is a quark isotopic operator acting on the isospin

indices of the quark 3. It is sandwiched with the isotopic spinors in (86) or in (87). Then we get:

$$
\left\langle\chi^{\prime(0)}|I| \chi^{(0)}\right\rangle=\sum_{\tau_{1}^{\prime} \tau_{1} \tau_{2} \tau_{3}}\left[\xi_{2}^{\tau_{2}} \tau_{y} \xi_{1}^{\tau_{1}}\right]\left[\xi_{N}^{\dagger \tau^{\prime}} \xi_{3}^{\tau_{3}^{\prime}}\right] I_{\tau_{3}^{\prime} \tau_{3}}\left[\xi_{3}^{\dagger \tau_{3}} \xi_{N}^{\tau}\right]\left[\xi_{1}^{\dagger \tau_{1}} \tau_{y} \xi_{2}^{* \tau_{2}}\right]=\operatorname{Tr}\left[\tau_{y} \tau_{y}\right] I_{\tau^{\prime} \tau}=2 I_{\tau^{\prime} \tau}
$$

where $\tau, \tau^{\prime}$ are the nucleon isospin projections.

For other matrix elements we similarly get:

$$
\left\langle\chi^{\prime(1)}|I| \chi^{(1)}\right\rangle=2\left(\tau_{i} I \tau_{i}\right)_{\tau^{\prime} \tau}, \quad\left\langle\chi^{\prime(1)}|I| \chi^{(0)}\right\rangle=\left\langle\chi^{\prime(0)}|I| \chi^{(1)}\right\rangle=0 .
$$

For the isospin structure of the electromagnetic current, eq. (86), we find:

$$
\left\langle\chi^{(0)}|I| \chi^{(0)}\right\rangle=\left(\begin{array}{cc}
4 / 3 & 0 \\
0 & -2 / 3
\end{array}\right), \quad\left\langle\chi^{(1)}|I| \chi^{(1)}\right\rangle=\left(1-\tau_{z}\right)_{\tau^{\prime} \tau}=\left(\begin{array}{ll}
0 & 0 \\
0 & 2
\end{array}\right) .
$$

For the axial current $I=\vec{\tau}$ we obtain:

$$
\left\langle\chi^{\prime(0)}|I| \chi^{(0)}\right\rangle=2 \vec{\tau}_{\tau^{\prime} \tau}, \quad\left\langle\chi^{\prime(1)}|I| \chi^{(1)}\right\rangle=2\left(\tau_{i} \vec{\tau} \tau_{i}\right)_{\tau^{\prime} \tau}=-2 \vec{\tau}_{\tau^{\prime} \tau}
$$




\subsection{The spin matrix elements}

We calculate the spin matrix elements of the operator $J_{\rho}=\bar{u}^{\prime}\left(k_{3}\right) j_{\rho}^{(3)} u\left(k_{3}\right)$ in the example of the symmetric wave function (64). For the electromagnetic current: $j_{\rho}^{(3)}=\gamma_{\rho}$. For the axial current: $j_{\rho}^{(3)}=\gamma_{\rho} \gamma_{5}$.

The final wave function reads (compare with (64)):

$$
\begin{aligned}
\Psi_{S}^{\prime \dagger}= & \frac{\psi_{S}^{\prime *}}{\sqrt{72}} c_{1}^{\prime} c_{2}^{\prime} c_{3}^{\prime} c_{N}^{\prime}\left\{3\left[u\left(k_{2}\right) U_{c} \Pi_{-}^{\prime} \gamma_{5} \Pi_{+}^{\prime} u\left(k_{1}\right)\right]\left[\bar{u}\left(p^{\prime}\right) \Pi_{+}^{\prime} \Pi_{+}^{\prime} u\left(k_{3}^{\prime}\right)\right]\right. \\
& \left.-\left[u\left(k_{2}\right) U_{c} \Pi_{-}^{\prime} \gamma^{\mu} \Pi_{+}^{\prime} u\left(k_{1}\right)\right]\left[\bar{u}\left(p^{\prime}\right) \Pi_{+}^{\prime} \gamma_{5} \gamma_{\mu} \Pi_{+}^{\prime} u\left(k_{3}^{\prime}\right)\right]\left(\vec{\tau}_{21} \cdot \vec{\tau}_{N 3}\right)\right\} \\
\equiv & \Psi_{0}^{\prime \dagger}+\Psi_{1}^{\prime \dagger} .
\end{aligned}
$$

where

$$
\Pi_{ \pm}^{\prime}=\frac{\mathcal{M}^{\prime} \pm \hat{\mathcal{P}}^{\prime}}{2 \mathcal{M}^{\prime}}, \quad \mathcal{P}^{\prime}=p^{\prime}+\omega \tau^{\prime}=k_{1}+k_{2}+k_{3}^{\prime}, \quad \mathcal{M}^{\prime}=\sqrt{\mathcal{P}^{\prime 2}}
$$

and $c_{1,2,3}^{\prime}, c_{N}^{\prime}$ differ from $c_{1,2,3}, c_{N}$, eq. (16), by the replacement of the initial momenta by the final ones. Since, due to (86) and (87), the quark 3 only is chosen as the interacting one, the momenta of the quarks 1 and 2 remain unchanged. The wave function $\Psi_{S}^{\prime \dagger}$ is written for this case.

The matrix element can be represented as:

$$
\left\langle\Psi_{S}^{\prime \dagger}\left|J_{\rho}\right| \Psi_{S}\right\rangle=\left\langle\Psi_{0}^{\prime \dagger}\left|J_{\rho}\right| \Psi_{0}\right\rangle+\left\langle\Psi_{1}^{\prime \dagger}\left|J_{\rho}\right| \Psi_{1}\right\rangle
$$

The nondiagonal matrix elements between the states $\left\langle\Psi_{0}^{\prime \dagger}\right|$ and $\left|\Psi_{1}\right\rangle$ do not contribute because of the zero nondiagonal isospin matrix elements, eq. (89).

For the matrix element $\left\langle\Psi_{0}^{\prime}\left|J_{\rho}\right| \Psi_{0}\right\rangle$ we start with the expression in terms of the spinors (omitting for a moment any isospin factors):

$$
\begin{aligned}
\left\langle\Psi_{0}^{\prime}\left|J_{\rho}\right| \Psi_{0}\right\rangle & =\frac{9}{72} c \psi_{S}^{\prime *} \psi_{S} \sum_{\sigma_{1}, \sigma_{2}, \sigma_{3}, \sigma_{3}^{\prime}}\left[u^{\sigma_{2}}\left(k_{2}\right) U_{c} \gamma_{5} \Pi_{+}^{\prime} u^{\sigma_{1}}\left(k_{1}\right)\right]\left[\bar{u}^{\sigma_{1}}\left(k_{1}\right) \Pi_{+} \gamma_{5} U_{c} \bar{u}^{\sigma_{2}}\left(k_{2}\right)\right] \\
& \times\left[\bar{u}^{\sigma^{\prime}}\left(p^{\prime}\right) \Pi_{+}^{\prime} u^{\sigma_{3}^{\prime}}\left(k_{3}^{\prime}\right)\right]\left[\bar{u}^{\sigma_{3}^{\prime}}\left(k_{3}^{\prime}\right) j_{\rho}^{(3)} u^{\sigma_{3}}\left(k_{3}\right)\right]\left[\bar{u}^{\sigma_{3}}\left(k_{3}\right) \Pi_{+} u^{\sigma}(p)\right] / x_{3},
\end{aligned}
$$

where

$$
c=c_{1} c_{2} c_{3} c_{N} c_{1}^{\prime} c_{2}^{\prime} c_{3}^{\prime} c_{N}^{\prime}, \quad x_{3}=\omega \cdot k_{3} / \omega \cdot p .
$$

We take into account that $\sum_{\sigma} u_{\alpha^{\prime}}^{\sigma}(k) \bar{u}_{\alpha}^{\sigma}(k)=(\hat{k}+m)_{\alpha^{\prime} \alpha},\left(U_{c} \gamma_{5}\right)^{t}=-\gamma_{5} U_{c}=-U_{c} \gamma_{5}$ and $\gamma_{5} U_{c}(\hat{k}+m)^{t}=(\hat{k}+m) \gamma_{5} U_{c}$ and get:

$$
\begin{aligned}
\left\langle\Psi_{0}^{\prime}\left|J_{\rho}\right| \Psi_{0}\right\rangle & =\frac{9}{72} c \psi_{S}^{*} \psi_{S} \operatorname{Tr}\left[\Pi_{+}^{\prime}\left(\hat{k}_{1}+m\right) \Pi_{+}\left(\hat{k}_{2}+m\right)\right] \\
& \times\left[\bar{u}^{\sigma^{\prime}}\left(p^{\prime}\right) \Pi_{+}^{\prime}\left(\hat{k}_{3}^{\prime}+m\right) j_{\rho}^{(3)}\left(\hat{k}_{3}+m\right) \Pi_{+} u^{\sigma}(p)\right] / x_{3} .
\end{aligned}
$$

Similarly we get another matrix element:

$$
\begin{aligned}
\left\langle\Psi_{1}^{\prime}\left|J_{\rho}\right| \Psi_{1}\right\rangle & =\frac{1}{72} c \psi_{S}^{\prime *} \psi_{S} \operatorname{Tr}\left[\Pi_{+}^{\prime} \gamma_{5} \gamma^{\nu} \Pi_{+}^{\prime}\left(\hat{k}_{1}+m\right) \Pi_{+} \gamma^{\mu} \gamma_{5} \Pi_{+}\left(\hat{k}_{2}+m\right)\right] \\
& \left.\times \bar{u}^{\sigma^{\prime}}\left(p^{\prime}\right) \Pi_{+}^{\prime} \gamma_{5} \gamma_{\nu} \Pi_{+}^{\prime}\left(\hat{k}_{3}^{\prime}+m\right) j_{\rho}^{(3)}\left(\hat{k}_{3}+m\right) \Pi_{+} \gamma_{\mu} \gamma_{5} \Pi_{+} u^{\sigma}(p)\right] / x_{3}
\end{aligned}
$$


Taking into account the isospin factors (90), we find by this way the matrix elements of the nucleon electromagnetic current.

\section{For proton:}

$$
\left\langle p^{\prime}\left|J_{\rho}\right| p\right\rangle=3 \int\left\{\frac{4}{3}\left\langle\Psi_{0}^{\prime}\left|J_{\rho}\right| \Psi_{0}\right\rangle\right\} D
$$

Note that the matrix element $\left\langle\Psi_{1}^{\prime}\left|J_{\rho}\right| \Psi_{1}\right\rangle$ does not contribute here.

For neutron:

$$
\left\langle n^{\prime}\left|J_{\rho}\right| n\right\rangle=3 \int\left\{-\frac{2}{3}\left\langle\Psi_{0}^{\prime}\left|J_{\rho}\right| \Psi_{0}\right\rangle+2\left\langle\Psi_{1}^{\prime}\left|J_{\rho}\right| \Psi_{1}\right\rangle\right\} D .
$$

With the isospin factor (91) we get the matrix elements

\section{for the axial current:}

$$
\begin{aligned}
\left\langle N^{\prime}\left|J_{\rho}^{A}\right| N\right\rangle & =\vec{\tau} A_{\rho} \\
A_{\rho} & =3 \int\left\{2\left\langle\Psi_{0}^{\prime}\left|J_{\rho}\right| \Psi_{0}\right\rangle-2\left\langle\Psi_{1}^{\prime}\left|J_{\rho}\right| \Psi_{1}\right\rangle\right\} D .
\end{aligned}
$$

These expressions can be represented in the form:

$$
\left\langle N^{\prime}\left|J_{\rho}\right| N\right\rangle=\bar{u}^{\prime}\left(p^{\prime}\right) G_{\rho} u(p)
$$

and determine by this way the $4 \times 4$-matrix $G_{\rho}$. Then we find the matrix

$$
O_{\rho}=\left(\hat{p}^{\prime}+M\right) G_{\rho}(\hat{p}+M) /\left(4 M^{2}\right) .
$$

The electromagnetic form factors are expressed through $O_{\rho}$ by the formulas given in the paper [23].

\section{Some applications}

\subsection{The proton magnetic moment}

The nucleon electromagnetic vertex in the light-front dynamics, i.e., the matrix $G_{\rho}$ in (99), due to approximations, depends on the orientation of the light-front plane. In the covariant LFD this dependence is parametrized in terms of the four-vector $\omega$. The vertex contains the non-physical $\omega$-depending terms, namely [23]:

$$
G_{\rho}=F_{1} \gamma_{\rho}+\frac{i F_{2}}{2 M} \sigma_{\rho \nu} q^{\nu}+B_{1}\left(\frac{\hat{\omega}}{\omega \cdot p}-\frac{1}{(1+\eta) M}\right) P_{\rho}+B_{2} \frac{M}{\omega \cdot p} \omega_{\rho}+B_{3} \frac{M^{2}}{(\omega \cdot p)^{2}} \hat{\omega} \omega_{\rho} .
$$

The method of calculating $G_{\rho}$ is explained in the previous section. The physical form factors $F_{1}, F_{2}$ should be extracted from $G_{\rho}$ so to separate them from the nonphysical terms $B_{1-3}$. 
Usually the form factors are found from the plus-component of the current, corresponding to the contraction $G_{\rho} \omega^{\rho}$. In this way, the terms $B_{1-3}$ do not contribute to the charge form factor $G_{E}=F_{1}-\eta F_{2}$ (here $\eta=Q^{2} /\left(4 M^{2}\right)$ ). It is given by the formula [23]:

$$
G_{E}=\frac{M}{2(\omega \cdot p)} \operatorname{Tr}\left[O_{\rho} \omega^{\rho}\right] .
$$

With the matrix $O_{\rho}$ determined through (96), (99) and (100) one can easily check that the proton form factor $G_{E}(0)$ coincides with the normalization integral (67). With $O_{\rho}$ determined from (97) the neutron form factor $G_{E}(0)$ equals to zero.

However, the form factor $F_{2}$ calculated through the plus-component (we denote it as $F_{2}^{\prime}$ ) contains the contribution of $B_{1}$, namely:

$$
F_{2}^{\prime}=F_{2}+\frac{2}{1+\eta} B_{1}=\frac{M}{2 \eta(\omega \cdot p)} \operatorname{Tr}\left[O_{\rho} \omega^{\rho}\left(\hat{\omega} \frac{M}{\omega \cdot p}-1\right)\right] .
$$

The formula for the form factor $F_{2}$, separated from $B_{1}$, is more lengthy. It is given in [23].

The proton magnetic moment is $\mu_{p}=G_{M}(0)$ and the anomalous magnetic moment is $a=G_{M}(0)-1=F_{2}(0)$. We analyze two cases: $(i)$ the form factors calculated through the plus-component of the current, without separating the non-physical $\omega$-depending terms in the nucleon electromagnetic vertex (that in the covariant approach corresponds to the contraction $\left.G_{\rho} \omega^{\rho}\right)$; $(i i)$ with separating the $\omega$-depending terms. In the first case we find from (103) for the anomalous magnetic moment:

$$
a^{\prime}=\frac{2 M}{\mathcal{M}} \int\left[\frac{\left(1-x_{3}\right) \mathcal{M}\left(m+x_{3} \mathcal{M}\right)-\vec{R}_{\perp 3}^{2} / 2}{\left(m+x_{3} \mathcal{M}\right)^{2}+\vec{R}_{\perp 3}^{2}}\right] \psi_{S}^{2} D .
$$

The expression for $a^{\prime}$ is written in the variables explained above in the section 6.2. It coincides with one found in [9] (see also [11]). Note that in these papers it is result of taking into account the Melosh rotation matrices. In the covariant approach the spin rotations are included automatically.

In the case (ii), i.e., separating the $\omega$-dependent terms, we get:

$$
a=2 \int\left[\frac{\left(1-x_{3}\right)\left(m+x_{3} \mathcal{M}\right)^{2}-2 x_{3} \vec{R}_{\perp 3}^{2}}{2 x_{3}\left[\left(m+x_{3} \mathcal{M}\right)^{2}+\vec{R}_{\perp 3}^{2}\right]}\right] \psi_{S}^{2} D
$$

It differs from (104) by the relativistic corrections which can be important. It does not contain explicitly the nucleon mass. In the nonrelativistic limit $M=\mathcal{M}=3 m, x_{3}=1 / 3$, $\vec{R}_{\perp 3}=0$ we find: $a^{\prime}=a=2$.

Note that the above expressions, for example, eq. (102), contain the product of two traces, since according to (94) $O_{\rho}$ includes already the trace over the loop of the quarks 1 and 2. This factorization is due to the fact that after permutations we have reduced the wave function to the form with the contracted pairs of the spinors 1-2 and 3- $N$. Otherwise, instead of the product of traces we would get trace of the product of the larger number of the Dirac matrices. This would complicate the calculations considerably. 
Calculating, for example, eq. (104) through the trace (103), we get the expression in terms of the scalar products between the four-vectors entering in (103) (initial and final nucleon and quark momenta $p, p^{\prime}, k_{1}, k_{2}, k_{3}, k_{3}^{\prime}$ and also $\left.\omega, \mathcal{P}, \mathcal{P}^{\prime}\right)$. Then we express these scalar products in terms of the variables $\vec{R}_{\perp i}, \vec{R}_{\perp i}^{\prime}, x_{i}$. These three-body kinematical relations are given in appendix to the paper [23].

\subsection{The axial form factor}

The vertex function corresponding to the axial current in the impulse approximation is given by eq. (98). As in the case of the nucleon electromagnetic vertex, it also depends on the orientation of the light-front plane. In the particular case of $q=0$ it has the form:

$$
\begin{aligned}
A_{\rho} & =\bar{u}^{\prime}(p) G_{\rho}^{A} u(p) \\
G_{\rho}^{A} & =\left(g_{A}(0) \gamma_{\rho}+B_{14}(0) \frac{1}{\omega \cdot p} \hat{\omega} p_{\rho}+B_{3}(0) \frac{M^{2}}{(\omega \cdot p)^{2}} \hat{\omega} \omega_{\rho}\right) \gamma_{5}
\end{aligned}
$$

From eq. (106) we find:

$$
g_{A}(0)=\operatorname{Tr}\left\{O_{\rho}\left[\hat{\omega} p^{\rho} /(\omega \cdot p)-\hat{\omega} \omega^{\rho} M^{2} /(\omega \cdot p)^{2}-\gamma^{\rho}\right] \gamma_{5}\right\} / 4
$$

where $O_{\rho}$ is given by (100) at $p^{\prime}=p$ with the replacement $G_{\rho} \rightarrow G_{\rho}^{A}$. Since the expression (107), besides $\omega_{\rho}$, contains the contractions with $p^{\rho}$ and $\gamma^{\rho}$, this means that like in the case of $F_{2}$ the plus-component is not enough to find $g_{A}$.

However, following the standard procedure and contracting $A_{\rho}$ with $\omega_{\rho}$, we get:

$$
g_{A}^{\prime}(0)=g_{A}(0)+B_{14}(0)=\frac{M^{2}}{2(\omega \cdot p)^{2}} \operatorname{Tr}\left[O_{\rho} \omega^{\rho} \hat{\omega} \gamma_{5}\right]
$$

Finding $G_{\rho}^{A}$ from (98) and substituting it in $(100)$ we get $O_{\rho}$. Then by (108) we find:

$$
g_{A}^{\prime}(0)=\frac{5}{3} \int\left[\frac{\left(m+x_{3} \mathcal{M}\right)^{2}-\vec{R}_{\perp 3}^{2}}{\left(m+x_{3} \mathcal{M}\right)^{2}+\vec{R}_{\perp 3}^{2}}\right] \psi_{S}^{2} D .
$$

This expression coincides with one found the papers [9, 10] (see also [11]). Like the magnetic moment, in these papers it also results from the Melosh rotation matrices.

\footnotetext{
${ }^{1}$ In the paper 23 the quark 1 was considered as the interacting one, whereas in the present paper it is the quark 3. Therefore in the latter case instead of the formulas of 23]

$$
\vec{R}_{\perp 1}^{\prime}=\vec{R}_{\perp 1}+\left(1-x_{1}\right) \vec{\Delta}, \quad \vec{R}_{\perp 2,3}^{\prime}=\vec{R}_{\perp 2,3}-x_{2,3} \vec{\Delta}
$$

one should use

$$
\vec{R}_{\perp 1,2}^{\prime}=\vec{R}_{\perp 1,2}-x_{1,2} \vec{\Delta}, \quad \vec{R}_{\perp 3}^{\prime}=\vec{R}_{\perp 3}+\left(1-x_{3}\right) \vec{\Delta}
$$
}


Substituting $O_{\rho}$ in eq. (107) we get $g_{A}(0)$ separated from the nonphysical form factor $B_{14}(0)$ :

$$
g_{A}(0)=\frac{5}{3} \int\left[\frac{\left(m+x_{3} \mathcal{M}\right)\left(m^{2}+x_{3} m \mathcal{M}+\vec{R}_{\perp 3}^{2}\right)}{x_{3} M\left[\left(m+x_{3} \mathcal{M}\right)^{2}+\vec{R}_{\perp 3}^{2}\right]}\right] \psi_{S}^{2} D .
$$

In the nonrelativistic limit we find: $g_{A}^{\prime}(0)=g_{A}(0)=5 / 3$.

\section{Conclusion}

We have established the general spin structure of the nucleon wave function in $3 q$-model. It contains sixteen spin components forming the full basis for decomposition of the wave function. After taking into account the isospin, the total number of all the scalar functions $\psi_{S}, \psi_{A}, \psi_{1,2}$, which are the coefficients at the front of all the structures, namely, the momentum-independent ones, sect. 6, the structures generated by the symmetric tensors, appendix E, and, at last, the structures generated by the antisymmetric tensors, appendix F, is 32. This just corresponds to 16 functions for proton and 16 for neutron.

We give recipe representing all these structures in the explicitly covariant form. After that the calculations of the form factors are the straightforward, through the standard routine of the Dirac matrices and of the trace techniques, like in the case of the Feynman approach. This fact is the strong advantage of the explicitly covariant version of LFD.

Among four spin structures independent of momenta there is one symmetric structure, eq. (48). Namely it, multiplied by the Melosh matrices, with the function $\psi_{S}$ approximated by the S-wave, is often used for calculating the nucleon properties (form factors, magnetic moments, etc.). Represented in the covariant form by eq. (64), it does not require any Melosh matrices. Our calculations by this way of the proton anomalous magnetic moment and of the nucleon axial form factor, with the same wave function eq. (48), like in the paper [9], but represented covariantly, gives, of course, the same result as in 9].

Separating the $\omega$-dependent terms in the vector and axial vertices, but still keeping only one spin structure, we get different results. We believe that in order to make comparison with experiment, one should investigate the influence of other components, including the new ones. Some of them may be important. One can either introduce these components phenomenologically and see their value from fit of the experimental data, or try to estimate them in an appropriate quark dynamics. Even in the deuteron wave function we found [22] the component (so called $f_{5}$ ) which dominates in moderately relativistic region over all other components, including S- and D-waves. The same can take place in the nucleon wave function.

\section{Acknowledgement}

The author is sincerely grateful to M. Beyer, V.M. Kolybasov, J.-F. Mathiot and H.J. Weber for stimulating discussions and valuable remarks. 


\section{A The Dirac matrices and the spinors}

We give below the explicit form of the Dirac matrices and the spinors in the standard representation.

The nucleon spinor has the form:

$$
u^{\sigma}(p)=\sqrt{\varepsilon_{p}+M}\left(\begin{array}{c}
1 \\
\frac{\vec{\sigma} \cdot \vec{p}}{\left(\varepsilon_{p}+M\right)}
\end{array}\right) w_{N}^{\sigma}
$$

where $w_{N}^{\sigma}$ is the two-component spinor and $\varepsilon_{p}=\sqrt{\vec{p}^{2}+M^{2}}$.

The spinor $\bar{u}\left(k_{1}\right)$ of the quark 1 reads:

$$
\bar{u}^{\sigma_{1}}\left(k_{1}\right)=u^{\dagger \sigma_{1}}(k) \gamma^{0}=\sqrt{\varepsilon_{k_{1}}+m} w_{1}^{\dagger \sigma_{1}}\left(1,-\frac{\vec{\sigma} \cdot \vec{k}_{1}}{\left(\varepsilon_{k_{1}}+m\right)}\right)
$$

and similarly for $\bar{u}\left(k_{3}\right)$.

For the quark 2 we use the charge conjugated spinor:

$$
U_{c} \bar{u}^{t}\left(k_{2}\right)=\sqrt{\varepsilon_{k_{2}}+m}\left(\begin{array}{c}
\frac{-\vec{\sigma} \cdot \vec{k}_{2}}{\left(\varepsilon_{k_{2}}+m\right)} \\
-1
\end{array}\right) \sigma_{y} w_{2}^{*}
$$

The charge conjugation matrix $U_{c}=\gamma^{2} \gamma^{0}$ :

$$
U_{c}=\left(\begin{array}{cc}
0 & -\sigma_{y} \\
-\sigma_{y} & 0
\end{array}\right)
$$

The Dirac matrices:

$$
\gamma^{0}=\left(\begin{array}{rr}
1 & 0 \\
0 & -1
\end{array}\right), \quad \vec{\gamma}=\left(\begin{array}{rr}
0 & \vec{\sigma} \\
-\vec{\sigma} & 0
\end{array}\right), \quad \gamma_{5}=-i \gamma^{0} \gamma^{1} \gamma^{2} \gamma^{3}=\left(\begin{array}{rr}
0 & -1 \\
-1 & 0
\end{array}\right)
$$

where $\vec{\sigma}$ are the Pauli matrices.

\section{B Relation between the tensors}

The relation between the symmetric traceless tensors of the second rank, defined by (20), has the form:

$$
\begin{aligned}
t^{i j} \equiv \quad & \left(1-\cos ^{2} \theta_{2 n}\right) T_{11}^{i j} / \vec{q}_{1}^{2}+\left(1-\cos ^{2} \theta_{1 n}\right) T_{22}^{i j} / \vec{q}_{2}^{2}+\left(1-\cos ^{2} \theta_{12}\right) T_{n n}^{i j} \\
- & \left(\cos \theta_{12}-\cos \theta_{1 n} \cos \theta_{2 n}\right) T_{12}^{i j} /\left(\left|\vec{q}_{1}\right|\left|\vec{q}_{2}\right|\right) \\
- & \left(\cos \theta_{1 n}-\cos \theta_{12} \cos \theta_{2 n}\right) T_{1 n}^{i j} /\left|\vec{q}_{1}\right| \\
- & \left(\cos \theta_{2 n}-\cos \theta_{12} \cos \theta_{1 n}\right) T_{2 n}^{i j} /\left|\vec{q}_{2}\right| \equiv 0 .
\end{aligned}
$$


where

$$
\cos \theta_{12}=\overrightarrow{q_{1}} \cdot \overrightarrow{q_{2}} /\left(\left|\vec{q}_{1}\right|\left|\overrightarrow{q_{2}}\right|\right), \quad \cos \theta_{1 n}=\overrightarrow{q_{1}} \cdot \vec{n} /\left|\overrightarrow{q_{1}}\right|, \quad \cos \theta_{2 n}=\overrightarrow{q_{2}} \cdot \vec{n} /\left|\overrightarrow{q_{2}}\right| .
$$

In order to prove (115), it is enough to check that:

$$
\begin{aligned}
& q_{1}^{i} t_{i j} q_{1}^{j}=0, \quad q_{2}^{i} t_{i j} q_{2}^{j}=0, \quad n^{i} t_{i j} n^{j}=0, \\
& q_{1}^{i} t_{i j} q_{2}^{j}=0, \quad q_{1}^{i} t_{i j} n^{j}=0, \quad q_{2}^{i} t_{i j} n^{j}=0 .
\end{aligned}
$$

Since any vectors $\vec{a}, \vec{b}$ can be decomposed in terms of $\overrightarrow{q_{1}}, \overrightarrow{q_{2}}, \vec{n}$, e.g.:

$$
\vec{a}=c_{1} \overrightarrow{q_{1}}+c_{2} \overrightarrow{q_{2}}+c_{3} \vec{n}
$$

from (116) it follows that $a^{i} t_{i j} b^{j} \equiv 0$ for arbitrary $\vec{a}, \vec{b}$. This means that

$$
t_{i j} \equiv 0
$$

The identity (115) allows to choose as a basis any five tensors. We exclude the tensor $T_{n n}^{i j}$.

\section{Relation between the spin structures}

It is not difficult to establish the following identities:

$$
\begin{array}{llll}
\left(\vec{\sigma} \cdot \vec{q}_{1}\right)\left(\vec{n} \cdot\left[\vec{q}_{1} \times \vec{q}_{2}\right]\right)=\vec{\sigma} \cdot\left[\vec{q}_{1} \times \vec{q}_{2}\right]\left(\overrightarrow{q_{1}} \cdot \vec{n}\right) & -\vec{\sigma} \cdot\left[\vec{q}_{1} \times \vec{n}\right]\left(\vec{q}_{1} \cdot \overrightarrow{q_{2}}\right) & +\vec{\sigma} \cdot\left[\vec{q}_{2} \times \vec{n}\right] \vec{q}_{1}^{2} \\
\left(\vec{\sigma} \cdot \vec{q}_{2}\right)\left(\vec{n} \cdot\left[\vec{q}_{1} \times \vec{q}_{2}\right]\right)=\vec{\sigma} \cdot\left[\vec{q}_{1} \times \vec{q}_{2}\right]\left(\overrightarrow{q_{2}} \cdot \vec{n}\right) & -\vec{\sigma} \cdot\left[\vec{q}_{1} \times \vec{n}\right] \vec{q}_{2}^{2} & +\vec{\sigma} \cdot\left[\vec{q}_{2} \times \vec{n}\right]\left(\vec{q}_{1} \cdot \vec{q}_{2}\right) \\
\left(\vec{\sigma} \cdot \overrightarrow{q_{3}}\right)\left(\vec{n} \cdot\left[\vec{q}_{1} \times \vec{q}_{2}\right]\right)=\vec{\sigma} \cdot\left[\overrightarrow{q_{1}} \times \overrightarrow{q_{2}}\right] & -\vec{\sigma} \cdot\left[\vec{q}_{1} \times \vec{n}\right]\left(\overrightarrow{q_{2}} \cdot \vec{n}\right) & +\vec{\sigma} \cdot\left[\overrightarrow{q_{2}} \times \vec{n}\right]\left(\overrightarrow{q_{1}} \cdot \vec{n}\right)
\end{array}
$$

where $\vec{\sigma}$ is arbitrary vector.

The reverse expressions have the form:

$$
\begin{aligned}
\vec{\sigma} \cdot\left[\vec{q}_{1} \times \vec{q}_{2}\right] & =\left(\vec{n} \cdot\left[\vec{q}_{1} \times \vec{q}_{2}\right]\right)\left\{-\left(\vec{\sigma} \cdot \vec{q}_{1}\right)\left[\vec{q}_{2}^{2}\left(\vec{q}_{1} \cdot \vec{n}\right)-\left(\vec{q}_{1} \cdot \vec{q}_{2}\right)\left(\vec{q}_{2} \cdot \vec{n}\right)\right]\right. \\
& \left.-\left(\vec{\sigma} \cdot \vec{q}_{2}\right)\left[\vec{q}_{1}^{2}\left(\vec{q}_{2} \cdot \vec{n}\right)-\left(\vec{q}_{1} \cdot \vec{q}_{2}\right)\left(\overrightarrow{q_{1}} \cdot \vec{n}\right)\right]+(\vec{\sigma} \cdot \vec{n})\left[\vec{q}_{1}^{2} \vec{q}_{2}^{2}-\left(\vec{q}_{1} \cdot \vec{q}_{2}\right)^{2}\right]\right\} / d \\
\vec{\sigma} \cdot\left[\vec{q}_{1} \times \vec{n}\right] & =\left(\vec{n} \cdot\left[\vec{q}_{1} \times \vec{q}_{2}\right]\right)\left\{\left(\vec{\sigma} \cdot \vec{q}_{1}\right)\left[\vec{q}_{1} \cdot \vec{q}_{2}-\left(\overrightarrow{q_{1}} \cdot \vec{n}\right)\left(\overrightarrow{q_{2}} \cdot \vec{n}\right)\right]\right. \\
& \left.-\left(\vec{\sigma} \cdot \vec{q}_{2}\right)\left[\vec{q}_{1}^{2}-\left(\overrightarrow{q_{1}} \cdot \vec{n}\right)^{2}\right]+(\vec{\sigma} \cdot \vec{n})\left[\vec{q}_{1}^{2}\left(\vec{q}_{2} \cdot \vec{n}\right)-\left(\vec{q}_{1} \cdot \vec{q}_{2}\right)\left(\vec{q}_{1} \cdot \vec{n}\right)\right]\right\} / d \\
\vec{\sigma} \cdot\left[\vec{q}_{2} \times \vec{n}\right] & =\left(\vec{n} \cdot\left[\vec{q}_{1} \times \vec{q}_{2}\right]\right)\left\{\left(\vec{\sigma} \cdot \vec{q}_{1}\right)\left[\vec{q}_{2}^{2}-\left(\overrightarrow{q_{2}} \cdot \vec{n}\right)^{2}\right]\right. \\
& \left.-\left(\vec{\sigma} \cdot \vec{q}_{2}\right)\left[\vec{q}_{1} \cdot \vec{q}_{2}-\left(\overrightarrow{q_{1}} \cdot \vec{n}\right)\left(\vec{q}_{2} \cdot \vec{n}\right)\right]-(\vec{\sigma} \cdot \vec{n})\left[\vec{q}_{2}^{2}\left(\vec{q}_{1} \cdot \vec{n}\right)-\left(\vec{q}_{1} \cdot \vec{q}_{2}\right)\left(\overrightarrow{q_{2}} \cdot \vec{n}\right)\right]\right\} / d
\end{aligned}
$$

where

$$
d=\left(\vec{n} \cdot\left[\vec{q}_{1} \times \vec{q}_{2}\right]\right)^{2}=\vec{q}_{1}^{2} \vec{q}_{2}^{2}-\vec{q}_{1}^{2}\left(\overrightarrow{q_{2}} \cdot \vec{n}\right)^{2}-\vec{q}_{2}^{2}\left(\overrightarrow{q_{1}} \cdot \vec{n}\right)^{2}-\left(\vec{q}_{1} \cdot \vec{q}_{2}\right)^{2}+2\left(\vec{q}_{1} \cdot \vec{q}_{2}\right)\left(\overrightarrow{q_{1}} \cdot \vec{n}\right)\left(\overrightarrow{q_{2}} \cdot \vec{n}\right)
$$

Due to these identities the set of the structures (11-16) of (24) is equivalent to the set formed by the structures $\left(\vec{\sigma}_{12} \cdot \overrightarrow{q_{1}}\right)\left(\vec{n} \cdot\left[\overrightarrow{q_{1}} \times \overrightarrow{q_{2}}\right]\right),\left(\overrightarrow{\sigma_{12}} \cdot \overrightarrow{q_{2}}\right)\left(\vec{n} \cdot\left[\overrightarrow{q_{1}} \times \overrightarrow{q_{2}}\right]\right)$, etc. 


\section{The Fierz identities for the Pauli matrices}

To establish the permutation properties (43) and (75) of the spin operators relative to the permutation $P_{13}$, we use the following Fierz identities:

$$
\begin{aligned}
P_{13} 1_{12} 1_{3 N}=1_{32} 1_{1 N} & =\frac{1}{2} 1_{12} 1_{3 N}+\frac{1}{2} \vec{\sigma}_{12} \cdot \vec{\sigma}_{3 N}, \\
P_{13} 1_{12} \sigma_{3 N}^{i}=1_{32} \sigma_{1 N}^{i} & =\frac{1}{2} 1_{12} \sigma_{3 N}^{i}+\frac{1}{2} \sigma_{12}^{i} 1_{3 N}-\frac{i}{2} \epsilon_{i k l} \sigma_{12}^{k} \sigma_{3 N}^{l}, \\
P_{13} \sigma_{12}^{i} 1_{3 N}=\sigma_{32}^{i} 1_{1 N} & =\frac{1}{2} 1_{12} \sigma_{3 N}^{i}+\frac{1}{2} \sigma_{12}^{i} 1_{3 N}+\frac{i}{2} \epsilon_{i k l} \sigma_{12}^{k} \sigma_{3 N}^{l}, \\
P_{13} \sigma_{12}^{i} \sigma_{3 N}^{j}=\sigma_{32}^{i} \sigma_{1 N}^{j} & =\frac{1}{2} \delta_{i j} 1_{12} 1_{3 N}+\frac{i}{2} \epsilon_{i j l} 1_{12} \sigma_{3 N}^{l}-\frac{i}{2} \epsilon_{i j l} \sigma_{12}^{l} 1_{3 N} \\
& -\frac{1}{2} \delta_{i j} \vec{\sigma}_{12} \cdot \vec{\sigma}_{3 N}+\frac{1}{2} \sigma_{12}^{i} \sigma_{3 N}^{j}+\frac{1}{2} \sigma_{12}^{j} \sigma_{3 N}^{i} .
\end{aligned}
$$

From (119) it follows:

$$
P_{13} \vec{\sigma}_{12} \cdot \vec{\sigma}_{3 N}=\vec{\sigma}_{32} \cdot \vec{\sigma}_{1 N}=\frac{3}{2} 1_{12} 1_{3 N}-\frac{1}{2} \vec{\sigma}_{12} \cdot \vec{\sigma}_{3 N}
$$

Under the permutation $P_{23}$ the operators are transformed as follows:

$$
\begin{aligned}
P_{23} 1_{12} 1_{3 N}=1_{13} 1_{2 N}= & \frac{1}{2} 1_{12} 1_{3 N}-\frac{1}{2} \vec{\sigma}_{12} \cdot \vec{\sigma}_{3 N}, \\
P_{23} 1_{12} \sigma_{3 N}^{i}=1_{13} \sigma_{2 N}^{i}= & \frac{1}{2} 1_{12} \sigma_{3 N}^{i}-\frac{1}{2} \sigma_{12}^{i} 1_{3 N}+\frac{i}{2} \epsilon_{i k l} \sigma_{12}^{k} \sigma_{3 N}^{l}, \\
P_{23} \sigma_{12}^{i} 1_{3 N}=\sigma_{13}^{i} 1_{2 N}= & -\frac{1}{2} 1_{12} \sigma_{3 N}^{i}+\frac{1}{2} \sigma_{12}^{i} 1_{3 N}+\frac{i}{2} \epsilon_{i k l} \sigma_{12}^{k} \sigma_{3 N}^{l}, \\
P_{23} \sigma_{12}^{i} \sigma_{3 N}^{j}=\sigma_{13}^{i} \sigma_{2 N}^{j}= & -\frac{1}{2} \delta_{i j} 1_{12} 1_{3 N}-\frac{i}{2} \epsilon_{i j l} 1_{12} \sigma_{3 N}^{l} \cdot-\frac{i}{2} \epsilon_{i j l} \sigma_{12}^{l} 1_{3 N} \\
& -\frac{1}{2} \delta_{i j} \vec{\sigma}_{12} \cdot \vec{\sigma}_{3 N}+\frac{1}{2} \sigma_{12}^{i} \sigma_{3 N}^{j}+\frac{1}{2} \sigma_{12}^{j} \sigma_{3 N}^{i} .
\end{aligned}
$$

From (121) it follows:

$$
P_{23} \vec{\sigma}_{12} \cdot \vec{\sigma}_{3 N}=\vec{\sigma}_{13} \cdot \vec{\sigma}_{2 N}=-\frac{3}{2} 1_{12} 1_{3 N}-\frac{1}{2} \vec{\sigma}_{12} \cdot \vec{\sigma}_{3 N}
$$

The permutation $P_{12}$ is evident:

$$
P_{12} 1_{12}=1_{21}=-1_{12}, \quad P_{12} \vec{\sigma}_{12}=\vec{\sigma}_{21}=\vec{\sigma}_{12} .
$$

A set of the Fierz identities for the Dirac matrices is given in [5]. 


\section{E Wave functions generated by the symmetric ten- sor structures}

Below we give the structures generated by the symmetric tensors, as described in sect. 7.1. The diagram shown below summarizes all the ways of generating the nucleon wave function $\Psi_{S}$ from the functions of different symmetries.

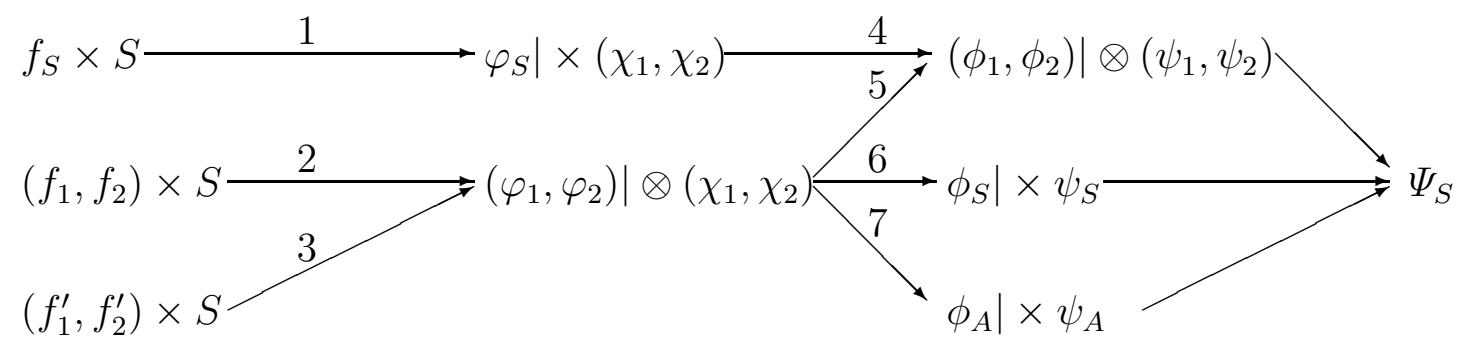

For example, the path $2 \rightarrow 5$ means that from the doublet $\left(f_{1}, f_{2}\right)$ multiplied by the symmetric spin tensor $S$ we obtain the doublet $\left(\varphi_{1}, \varphi_{2}\right)$. Then with the isospin doublet $\left(\chi_{1}, \chi_{2}\right)$, by eq. $\left.38, \mathrm{~s}\right)$, we construct the spin-isospin doublet $\left(\phi_{1}, \phi_{2}\right)$. Then with the momentum scalar functions $\left(\psi_{1}, \psi_{2}\right)$, by (41), we get $\Psi_{S}$ given by (125). Seven paths connecting the initial states at the left of the diagram with the final one $\Psi_{S}$ correspond to seven functions given below.

Before any function we indicate in more detail, how the given structure is obtained. For example, the notation above eq. (126)

$$
2 \rightarrow 6: \quad\left(f_{1}, f_{2}\right) \times \stackrel{(71)}{S} \stackrel{(29)}{\longrightarrow}\left(\varphi_{1}, \varphi_{2}\right)\left|\otimes\left(\chi_{1}, \chi_{2}\right) \stackrel{(16)}{\longrightarrow} \phi_{S}\right| \times \psi_{S} \stackrel{(39)}{\longrightarrow} \stackrel{\sqrt{126})}{\Psi_{S}}
$$

means that eq. (126) is obtained by the path $2 \rightarrow 6$. Namely, the doublet $(70, \mathrm{a})$, formed by the momentum depending functions $\left(f_{1}, f_{2}\right)$, is multiplied by the symmetric spin tensor $S$, defined by (71), and gives by (72) the spin doublet $\left(\varphi_{1}, \varphi_{2}\right)$ (the line 2 on the diagram). Then, together with the isospin doublet $\left(\chi_{1}, \chi_{2}\right)$, defined by (46), by means of eq. (36), it gives the symmetric spin-isospin function $\phi_{S}$ (the line 6 on the diagram). Multiplied by $\psi_{S}$ by eq. (39), it gives the total symmetric nucleon wave function $\Psi_{S}$, eq. (126). All other notations are similar.

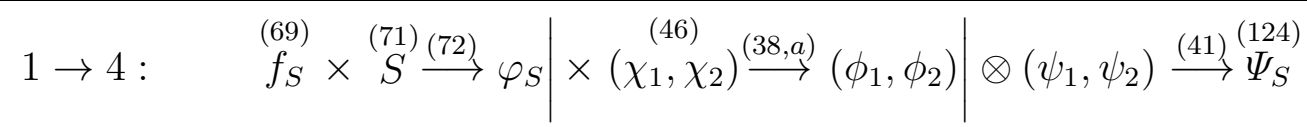

$$
\begin{aligned}
& \Psi_{S}=\psi_{1}\left[\left(\vec{q}_{1} \cdot \vec{\sigma}_{12}\right)\left(\vec{q}_{1} \cdot \vec{\sigma}_{3 N}\right)-\frac{1}{3} \vec{q}_{1}^{2}\left(\vec{\sigma}_{12} \cdot \vec{\sigma}_{3 N}\right)+\left(\vec{q}_{2} \cdot \vec{\sigma}_{12}\right)\left(\vec{q}_{2} \cdot \vec{\sigma}_{3 N}\right)-\frac{1}{3} \vec{q}_{2}^{2}\left(\vec{\sigma}_{12} \cdot \vec{\sigma}_{3 N}\right)\right. \\
& \left.+\quad\left(\vec{q}_{3} \cdot \vec{\sigma}_{12}\right)\left(\vec{q}_{3} \cdot \vec{\sigma}_{3 N}\right)-\frac{1}{3} \vec{q}_{3}^{2}\left(\vec{\sigma}_{12} \cdot \vec{\sigma}_{3 N}\right)\right]\left(\vec{\tau}_{12} \cdot \vec{\tau}_{3 N}-3\right) \\
& +\psi_{2}\left[\left(\vec{q}_{1} \cdot \vec{\sigma}_{12}\right)\left(\vec{q}_{1} \cdot \vec{\sigma}_{3 N}\right)-\frac{1}{3} \vec{q}_{1}^{2}\left(\vec{\sigma}_{12} \cdot \vec{\sigma}_{3 N}\right)+\left(\vec{q}_{2} \cdot \vec{\sigma}_{12}\right)\left(\vec{q}_{2} \cdot \vec{\sigma}_{3 N}\right)-\frac{1}{3} \vec{q}_{2}^{2}\left(\vec{\sigma}_{12} \cdot \vec{\sigma}_{3 N}\right)\right. \\
& \left.+\quad\left(\vec{q}_{3} \cdot \vec{\sigma}_{12}\right)\left(\vec{q}_{3} \cdot \vec{\sigma}_{3 N}\right)-\frac{1}{3} \vec{q}_{3}^{2}\left(\vec{\sigma}_{12} \cdot \vec{\sigma}_{3 N}\right)\right]\left(\vec{\tau}_{12} \cdot \vec{\tau}_{3 N}+3\right)
\end{aligned}
$$




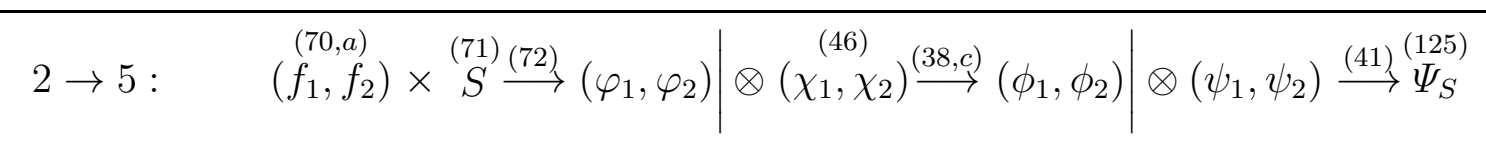

$$
\begin{aligned}
& \Psi_{S}=\psi_{1}\left\{\left[\left(\vec{q}_{1} \cdot \vec{\sigma}_{12}\right)\left(\vec{q}_{1} \cdot \vec{\sigma}_{3 N}\right)-\frac{1}{3} \vec{q}_{1}^{2}\left(\vec{\sigma}_{12} \cdot \vec{\sigma}_{3 N}\right)\right]\left(\vec{\tau}_{12} \cdot \vec{\tau}_{3 N}-3\right)\right. \\
& -2\left[\left(\vec{q}_{2} \cdot \vec{\sigma}_{12}\right)\left(\vec{q}_{2} \cdot \vec{\sigma}_{3 N}\right)-\frac{1}{3} \vec{q}_{2}^{2}\left(\vec{\sigma}_{12} \cdot \vec{\sigma}_{3 N}\right)\right] \vec{\tau}_{12} \cdot \vec{\tau}_{3 N} \\
& \left.+\left[\left(\vec{q}_{3} \cdot \vec{\sigma}_{12}\right)\left(\vec{q}_{3} \cdot \vec{\sigma}_{3 N}\right)-\frac{1}{3} \vec{q}_{3}^{2}\left(\vec{\sigma}_{12} \cdot \vec{\sigma}_{3 N}\right)\right]\left(\vec{\tau}_{12} \cdot \vec{\tau}_{3 N}+3\right)\right\} \\
& +\psi_{2}\left\{-2\left[\left(\vec{q}_{1} \cdot \vec{\sigma}_{12}\right)\left(\vec{q}_{1} \cdot \vec{\sigma}_{3 N}\right)-\frac{1}{3} \vec{q}_{1}^{2}\left(\vec{\sigma}_{12} \cdot \vec{\sigma}_{3 N}\right)\right] \vec{\tau}_{12} \cdot \vec{\tau}_{3 N}\right. \\
& +\left[\left(\vec{q}_{2} \cdot \vec{\sigma}_{12}\right)\left(\vec{q}_{2} \cdot \vec{\sigma}_{3 N}\right)-\frac{1}{3} \vec{q}_{2}^{2}\left(\vec{\sigma}_{12} \cdot \vec{\sigma}_{3 N}\right)\right]\left(\vec{\tau}_{12} \cdot \vec{\tau}_{3 N}+3\right) \\
& \left.+\left[\left(\vec{q}_{3} \cdot \vec{\sigma}_{12}\right)\left(\vec{q}_{3} \cdot \vec{\sigma}_{3 N}\right)-\frac{1}{3} \vec{q}_{3}^{2}\left(\vec{\sigma}_{12} \cdot \vec{\sigma}_{3 N}\right)\right]\left(\vec{\tau}_{12} \cdot \vec{\tau}_{3 N}-3\right)\right\} \\
& \Psi_{S}=\psi_{S}\left\{\left[\left(\vec{q}_{1} \cdot \vec{\sigma}_{12}\right)\left(\vec{q}_{1} \cdot \vec{\sigma}_{3 N}\right)-\frac{1}{3} \vec{q}_{1}^{2}\left(\vec{\sigma}_{12} \cdot \vec{\sigma}_{3 N}\right)\right]\left(\vec{\tau}_{12} \cdot \vec{\tau}_{3 N}-3\right)\right. \\
& +\quad\left[\left(\vec{q}_{2} \cdot \vec{\sigma}_{12}\right)\left(\vec{q}_{2} \cdot \vec{\sigma}_{3 N}\right)-\frac{1}{3} \vec{q}_{2}^{2}\left(\vec{\sigma}_{12} \cdot \vec{\sigma}_{3 N}\right)\right]\left(\vec{\tau}_{12} \cdot \vec{\tau}_{3 N}+3\right) \\
& \left.-\quad 2\left[\left(\vec{q}_{3} \cdot \vec{\sigma}_{12}\right)\left(\vec{q}_{3} \cdot \vec{\sigma}_{3 N}\right)-\frac{1}{3} \vec{q}_{3}^{2}\left(\vec{\sigma}_{12} \cdot \vec{\sigma}_{3 N}\right)\right] \vec{\tau}_{12} \cdot \vec{\tau}_{3 N}\right\}
\end{aligned}
$$

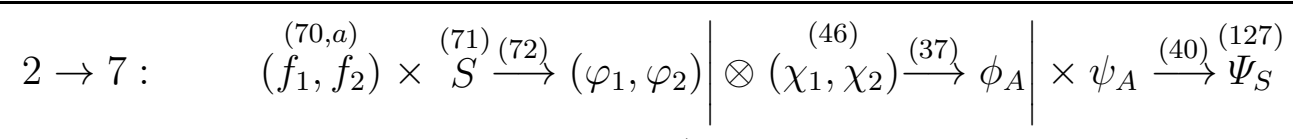

$$
\begin{aligned}
& \Psi_{S}=\psi_{A}\left\{\left[\left(\vec{q}_{1} \cdot \vec{\sigma}_{12}\right)\left(\vec{q}_{1} \cdot \vec{\sigma}_{3 N}\right)-\frac{1}{3} \vec{q}_{1}^{2}\left(\vec{\sigma}_{12} \cdot \vec{\sigma}_{3 N}\right)\right]\left(\vec{\tau}_{12} \cdot \vec{\tau}_{3 N}+1\right)\right. \\
& -\quad\left[\left(\vec{q}_{2} \cdot \vec{\sigma}_{12}\right)\left(\vec{q}_{2} \cdot \vec{\sigma}_{3 N}\right)-\frac{1}{3} \vec{q}_{2}^{2}\left(\vec{\sigma}_{12} \cdot \vec{\sigma}_{3 N}\right)\right]\left(\vec{\tau}_{12} \cdot \vec{\tau}_{3 N}-1\right) \\
& \left.-2\left[\left(\vec{q}_{3} \cdot \vec{\sigma}_{12}\right)\left(\vec{q}_{3} \cdot \vec{\sigma}_{3 N}\right)-\frac{1}{3} \vec{q}_{3}^{2}\left(\vec{\sigma}_{12} \cdot \vec{\sigma}_{3 N}\right)\right]\right\} \\
& 3 \rightarrow 5: \quad\left(f_{1}^{\prime}, f_{2}^{\prime}\right) \times \stackrel{(71)}{S} \stackrel{\sqrt[72]{\longrightarrow}}{\longrightarrow}\left(\varphi_{1}, \varphi_{2}\right)\left|\otimes\left(\chi_{1}, \chi_{2}\right) \stackrel{\sqrt{46}, c)}{\longrightarrow}\left(\phi_{1}, \phi_{2}\right)\right| \otimes\left(\psi_{1}, \psi_{2}\right) \stackrel{(41)}{\longrightarrow} \underset{\Psi_{S}}{(128)} \\
& \Psi_{S}=\psi_{1}\left\{2\left[\left(\vec{q}_{1} \cdot \vec{\sigma}_{12}\right)\left(\vec{n} \cdot \vec{\sigma}_{3 N}\right)+\left(\vec{n} \cdot \vec{\sigma}_{12}\right)\left(\vec{q}_{1} \cdot \vec{\sigma}_{3 N}\right)-\frac{2}{3}\left(\vec{q}_{1} \cdot \vec{n}\right)\left(\vec{\sigma}_{12} \cdot \vec{\sigma}_{3 N}\right)\right]\right. \\
& \left.+\quad\left[\left(\vec{q}_{2} \cdot \vec{\sigma}_{12}\right)\left(\vec{n} \cdot \vec{\sigma}_{3 N}\right)+\left(\vec{n} \cdot \vec{\sigma}_{12}\right)\left(\vec{q}_{2} \cdot \vec{\sigma}_{3 N}\right)-\frac{2}{3}\left(\vec{q}_{2} \cdot \vec{n}\right)\left(\vec{\sigma}_{12} \cdot \vec{\sigma}_{3 N}\right)\right]\left(\vec{\tau}_{12} \cdot \vec{\tau}_{3 N}+1\right)\right\} \\
& +\psi_{2}\left\{\left[\left(\vec{q}_{1} \cdot \vec{\sigma}_{12}\right)\left(\vec{n} \cdot \vec{\sigma}_{3 N}\right)+\left(\vec{n} \cdot \vec{\sigma}_{12}\right)\left(\vec{q}_{1} \cdot \vec{\sigma}_{3 N}\right)-\frac{2}{3}\left(\vec{q}_{1} \cdot \vec{n}\right)\left(\vec{\sigma}_{12} \cdot \vec{\sigma}_{3 N}\right)\right]\left(\vec{\tau}_{12} \cdot \vec{\tau}_{3 N}-1\right)\right. \\
& \left.-2\left[\left(\vec{q}_{2} \cdot \vec{\sigma}_{12}\right)\left(\vec{n} \cdot \vec{\sigma}_{3 N}\right)+\left(\vec{n} \cdot \vec{\sigma}_{12}\right)\left(\vec{q}_{2} \cdot \vec{\sigma}_{3 N}\right)-\frac{2}{3}\left(\vec{q}_{2} \cdot \vec{n}\right)\left(\vec{\sigma}_{12} \cdot \vec{\sigma}_{3 N}\right)\right]\right\}
\end{aligned}
$$




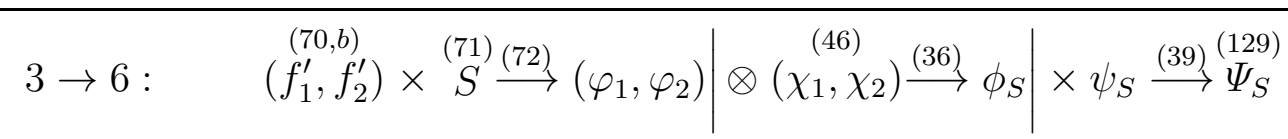

$$
\begin{aligned}
& \Psi_{S}=\psi_{S}\left\{\left[\left(\vec{q}_{1} \cdot \vec{\sigma}_{12}\right)\left(\vec{n} \cdot \vec{\sigma}_{3 N}\right)+\left(\vec{n} \cdot \vec{\sigma}_{12}\right)\left(\vec{q}_{1} \cdot \vec{\sigma}_{3 N}\right)-\frac{2}{3}\left(\overrightarrow{q_{1}} \cdot \vec{n}\right)\left(\vec{\sigma}_{12} \cdot \vec{\sigma}_{3 N}\right)\right]\left(\vec{\tau}_{12} \cdot \vec{\tau}_{3 N}-1\right)\right. \\
& \left.+\quad\left[\left(\vec{q}_{2} \cdot \vec{\sigma}_{12}\right)\left(\vec{n} \cdot \vec{\sigma}_{3 N}\right)+\left(\vec{n} \cdot \vec{\sigma}_{12}\right)\left(\vec{q}_{2} \cdot \vec{\sigma}_{3 N}\right)-\frac{2}{3}\left(\vec{q}_{2} \cdot \vec{n}\right)\left(\vec{\sigma}_{12} \cdot \vec{\sigma}_{3 N}\right)\right]\left(\vec{\tau}_{12} \cdot \vec{\tau}_{3 N}+1\right)\right\}
\end{aligned}
$$

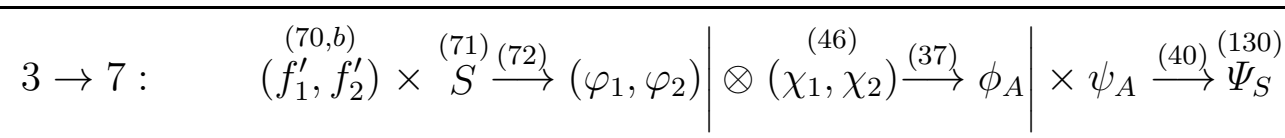

$$
\begin{aligned}
& \Psi_{S}=\psi_{A}\left\{\left[\left(\vec{q}_{1} \cdot \vec{\sigma}_{12}\right)\left(\vec{n} \cdot \vec{\sigma}_{3 N}\right)+\left(\vec{n} \cdot \vec{\sigma}_{12}\right)\left(\vec{q}_{1} \cdot \vec{\sigma}_{3 N}\right)-\frac{2}{3}\left(\vec{q}_{1} \cdot \vec{n}\right)\left(\vec{\sigma}_{12} \cdot \vec{\sigma}_{3 N}\right)\right]\left(\vec{\tau}_{12} \cdot \vec{\tau}_{3 N}+3\right)\right. \\
& \left.+\quad\left[\left(\vec{q}_{2} \cdot \vec{\sigma}_{12}\right)\left(\vec{n} \cdot \vec{\sigma}_{3 N}\right)+\left(\vec{n} \cdot \vec{\sigma}_{12}\right)\left(\vec{q}_{2} \cdot \vec{\sigma}_{3 N}\right)-\frac{2}{3}\left(\overrightarrow{q_{2}} \cdot \vec{n}\right)\left(\vec{\sigma}_{12} \cdot \vec{\sigma}_{3 N}\right)\right]\left(3-\vec{\tau}_{12} \cdot \vec{\tau}_{3 N}\right)\right\}
\end{aligned}
$$

\section{F Wave functions generated by the antisymmetric tensor structures}

As described in sect. 7.2, the antisymmetric tensors generate the functions given below. Like in appendix $\mathbb{E}$, the diagram shown below summarizes all the ways of generating the nucleon wave function $\Psi_{S}$ from the functions of different symmetries.

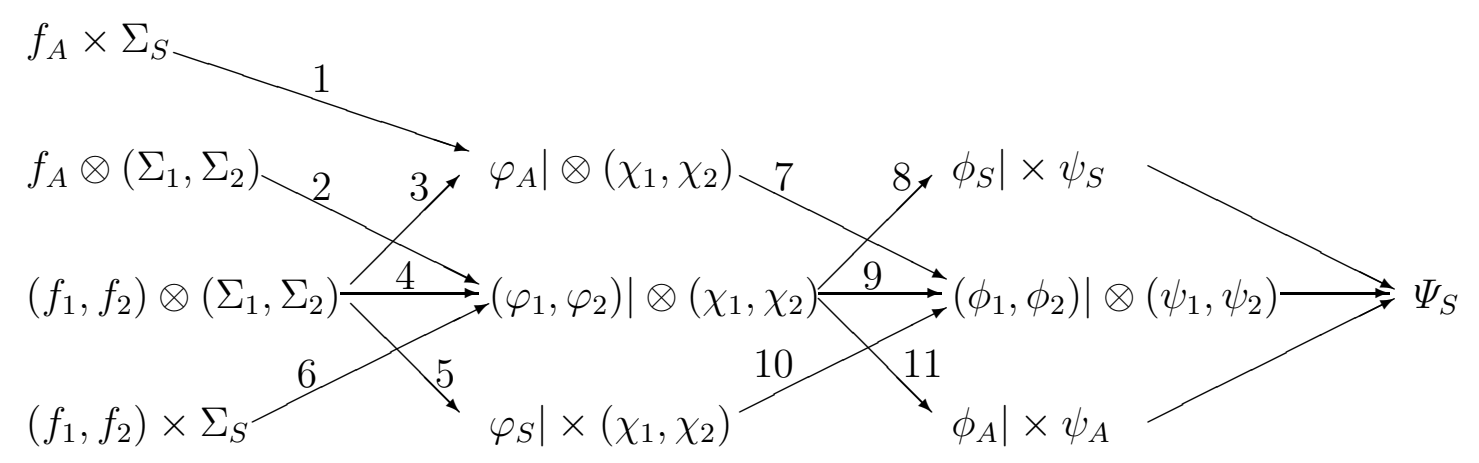

For example, the path $1 \rightarrow 7$ means that the antisymmetric function $f_{A}$ multiplied by the symmetric spin tensor $\Sigma_{S}$ gives the antisymmetric $\varphi_{A}$. Then with the isospin doublet $\left(\chi_{1}, \chi_{2}\right)$, by eq. (38,b), we construct the spin-isospin doublet $\left(\phi_{1}, \phi_{2}\right)$. Then with the momentum scalar functions $\left(\psi_{1}, \psi_{2}\right)$, by (41), we get $\Psi_{S}$ given by (131). given by (125). Twelve paths connecting the initial states at the left of the diagram with the final one $\Psi_{S}$ correspond to twelve functions given below.

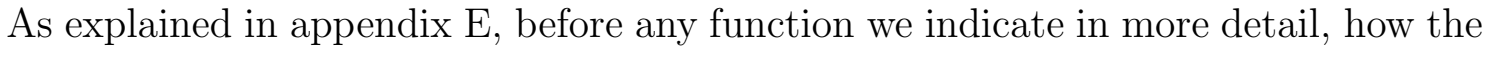


given structure is obtained.

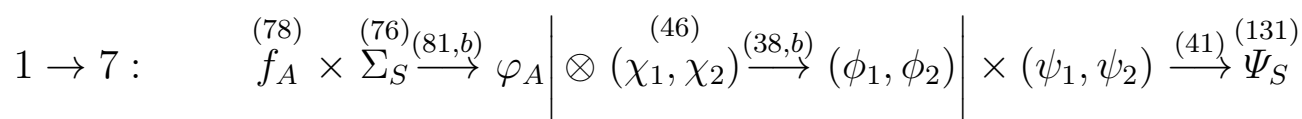

$$
\begin{aligned}
& \Psi_{S}=-\psi_{1}\left\{\left(\vec{q}_{1} \cdot \vec{\sigma}_{12}\right)\left(\vec{q}_{2} \cdot \vec{\sigma}_{3 N}\right)-\left(\vec{q}_{2} \cdot \vec{\sigma}_{12}\right)\left(\vec{q}_{1} \cdot \vec{\sigma}_{3 N}\right)-2 i \vec{\sigma}_{12} \cdot\left[\vec{q}_{1} \times \vec{q}_{2}\right]\right\}\left(1+\vec{\tau}_{12} \cdot \vec{\tau}_{3 N}\right) \\
& -\psi_{2}\left\{\left(\vec{q}_{1} \cdot \vec{\sigma}_{12}\right)\left(\vec{q}_{2} \cdot \vec{\sigma}_{3 N}\right)-\left(\vec{q}_{2} \cdot \vec{\sigma}_{12}\right)\left(\vec{q}_{1} \cdot \vec{\sigma}_{3 N}\right)-2 i \vec{\sigma}_{12} \cdot\left[\vec{q}_{1} \times \vec{q}_{2}\right]\right\}\left(1-\vec{\tau}_{12} \cdot \vec{\tau}_{3 N}\right)
\end{aligned}
$$

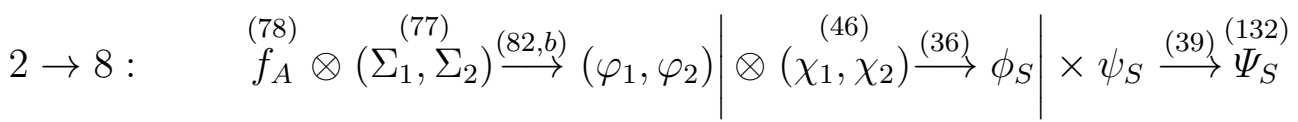

$$
\begin{aligned}
& \Psi_{S}=\psi_{S}\left\{\left(\vec{q}_{1} \cdot \vec{\sigma}_{12}\right)\left(\vec{q}_{2} \cdot \vec{\sigma}_{3 N}\right)-\left(\vec{q}_{2} \cdot \vec{\sigma}_{12}\right)\left(\vec{q}_{1} \cdot \vec{\sigma}_{3 N}\right)+i \vec{\sigma}_{12} \cdot\left[\vec{q}_{1} \times \vec{q}_{2}\right]\right. \\
& \left.-\quad i \vec{\sigma}_{3 N} \cdot\left[\vec{q}_{1} \times \vec{q}_{2}\right] \vec{\tau}_{12} \cdot \vec{\tau}_{3 N}\right\}
\end{aligned}
$$

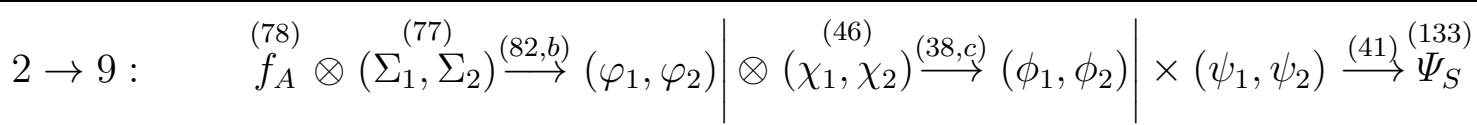

$$
\begin{aligned}
& \Psi_{S}=\psi_{1}\left\{\left[\left(\vec{q}_{1} \cdot \vec{\sigma}_{12}\right)\left(\vec{q}_{2} \cdot \vec{\sigma}_{3 N}\right)-\left(\vec{q}_{2} \cdot \vec{\sigma}_{12}\right)\left(\vec{q}_{1} \cdot \vec{\sigma}_{3 N}\right)+i \vec{\sigma}_{12} \cdot\left[\vec{q}_{1} \times \vec{q}_{2}\right]\right]\left(1-\vec{\tau}_{12} \cdot \vec{\tau}_{3 N}\right)\right. \\
& \left.+i \vec{\sigma}_{3 N} \cdot\left[\vec{q}_{1} \times \vec{q}_{2}\right]\left(3+\vec{\tau}_{12} \cdot \vec{\tau}_{3 N}\right)\right\} \\
& +\psi_{2}\left\{\left[\left(\vec{q}_{1} \cdot \vec{\sigma}_{12}\right)\left(\vec{q}_{2} \cdot \vec{\sigma}_{3 N}\right)-\left(\vec{q}_{2} \cdot \vec{\sigma}_{12}\right)\left(\vec{q}_{1} \cdot \vec{\sigma}_{3 N}\right)+i \vec{\sigma}_{12} \cdot\left[\vec{q}_{1} \times \vec{q}_{2}\right]\right]\left(1+\vec{\tau}_{12} \cdot \vec{\tau}_{3 N}\right)\right. \\
& \left.-i \vec{\sigma}_{3 N} \cdot\left[\vec{q}_{1} \times \vec{q}_{2}\right]\left(3-\vec{\tau}_{12} \cdot \vec{\tau}_{3 N}\right)\right\}
\end{aligned}
$$

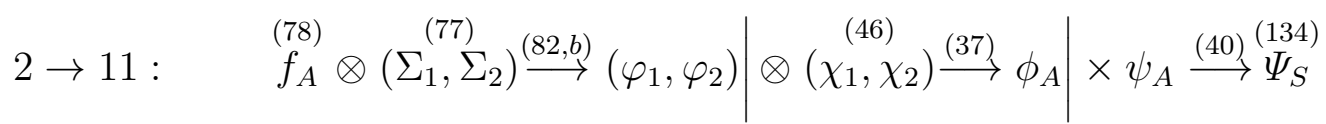$$
\Psi_{S}=\psi_{A}\left\{-\left[\left(\vec{q}_{1} \cdot \vec{\sigma}_{12}\right)\left(\vec{q}_{2} \cdot \vec{\sigma}_{3 N}\right)-\left(\vec{q}_{2} \cdot \vec{\sigma}_{12}\right)\left(\vec{q}_{1} \cdot \vec{\sigma}_{3 N}\right)+i \vec{\sigma}_{12} \cdot\left[\vec{q}_{1} \times \vec{q}_{2}\right]\right] \vec{\tau}_{12} \cdot \vec{\tau}_{3 N}\right.
$$

$$
\begin{aligned}
& \left.-\quad 3 i \vec{\sigma}_{3 N} \cdot\left[\vec{q}_{1} \times \vec{q}_{2}\right]\right\} \\
& 3 \rightarrow 7: \quad\left(f_{1}, f_{2}\right) \otimes\left(\Sigma_{1}, \Sigma_{2}\right) \stackrel{(\text { 国 }, a)}{\longrightarrow} \varphi_{A}\left|\otimes\left(\chi_{1}, \chi_{2}\right) \stackrel{(169), b)}{\longrightarrow}\left(\phi_{1}, \phi_{2}\right)\right| \times\left(\psi_{1}, \psi_{2}\right) \stackrel{(417)}{\longrightarrow} \stackrel{(135)}{\Psi_{S}} \\
& \Psi_{S}=\psi_{1}\left\{\left(\vec{q}_{1} \cdot \vec{\sigma}_{12}\right)\left(\vec{n} \cdot \vec{\sigma}_{3 N}\right)-\left(\vec{n} \cdot \vec{\sigma}_{12}\right)\left(\vec{q}_{1} \cdot \vec{\sigma}_{3 N}\right)-\left(\vec{q}_{2} \cdot \vec{\sigma}_{12}\right)\left(\vec{n} \cdot \vec{\sigma}_{3 N}\right)+\left(\vec{n} \cdot \vec{\sigma}_{12}\right)\left(\vec{q}_{2} \cdot \vec{\sigma}_{3 N}\right)\right. \\
& \left.+\quad i \vec{\sigma}_{12} \cdot\left[\vec{q}_{1} \times \vec{n}\right]+3 i \vec{\sigma}_{3 N} \cdot\left[\vec{q}_{1} \times \vec{n}\right]-i \vec{\sigma}_{12} \cdot\left[\vec{q}_{2} \times \vec{n}\right]+3 i \vec{\sigma}_{3 N} \cdot\left[\vec{q}_{2} \times \vec{n}\right]\right\}\left(1+\vec{\tau}_{12} \cdot \vec{\tau}_{3 N}\right) \\
& +\psi_{2}\left\{\left(\vec{q}_{1} \cdot \vec{\sigma}_{12}\right)\left(\vec{n} \cdot \vec{\sigma}_{3 N}\right)-\left(\vec{n} \cdot \vec{\sigma}_{12}\right)\left(\vec{q}_{1} \cdot \vec{\sigma}_{3 N}\right)-\left(\vec{q}_{2} \cdot \vec{\sigma}_{12}\right)\left(\vec{n} \cdot \vec{\sigma}_{3 N}\right)+\left(\vec{n} \cdot \vec{\sigma}_{12}\right)\left(\vec{q}_{2} \cdot \vec{\sigma}_{3 N}\right)\right. \\
& \left.+\quad i \vec{\sigma}_{12} \cdot\left[\vec{q}_{1} \times \vec{n}\right]+3 i \vec{\sigma}_{3 N} \cdot\left[\vec{q}_{1} \times \vec{n}\right]-i \vec{\sigma}_{12} \cdot\left[\vec{q}_{2} \times \vec{n}\right]+3 i \vec{\sigma}_{3 N} \cdot\left[\vec{q}_{2} \times \vec{n}\right]\right\}\left(1-\vec{\tau}_{12} \cdot \vec{\tau}_{3 N}\right)
\end{aligned}
$$

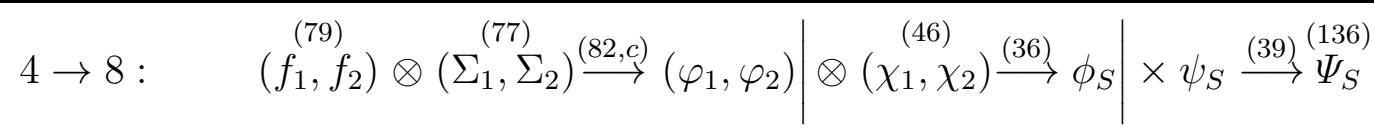

$$
\begin{aligned}
& \Psi_{S}=\psi_{S}\left\{-\left[\left(\vec{q}_{1} \cdot \vec{\sigma}_{12}\right)\left(\vec{n} \cdot \vec{\sigma}_{3 N}\right)-\left(\vec{n} \cdot \vec{\sigma}_{12}\right)\left(\vec{q}_{1} \cdot \vec{\sigma}_{3 N}\right)+i \vec{\sigma}_{12} \cdot\left[\vec{q}_{1} \times \vec{n}\right]\right]\left(1+\vec{\tau}_{12} \cdot \vec{\tau}_{3 N}\right)\right. \\
& +\quad\left[\left(\vec{q}_{2} \cdot \vec{\sigma}_{12}\right)\left(\vec{n} \cdot \vec{\sigma}_{3 N}\right)-\left(\vec{n} \cdot \vec{\sigma}_{12}\right)\left(\vec{q}_{2} \cdot \vec{\sigma}_{3 N}\right)+i \vec{\sigma}_{12} \cdot\left[\vec{q}_{2} \times \vec{n}\right]\right]\left(1-\vec{\tau}_{12} \cdot \vec{\tau}_{3 N}\right) \\
& \left.+\quad i \vec{\sigma}_{3 N} \cdot\left[\vec{q}_{1} \times \vec{n}\right]\left(3-\vec{\tau}_{12} \cdot \vec{\tau}_{3 N}\right)+i \vec{\sigma}_{3 N} \cdot\left[\vec{q}_{2} \times \vec{n}\right]\left(3+\vec{\tau}_{12} \cdot \vec{\tau}_{3 N}\right)\right\}
\end{aligned}
$$

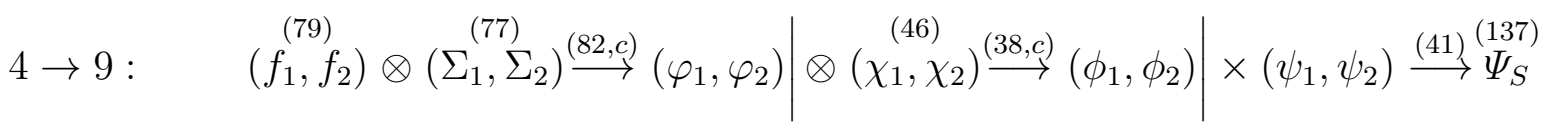

$$
\begin{aligned}
& \Psi_{S}=\psi_{1}\left\{\left[\left(\vec{q}_{1} \cdot \vec{\sigma}_{12}\right)\left(\vec{n} \cdot \vec{\sigma}_{3 N}\right)-\left(\vec{n} \cdot \vec{\sigma}_{12}\right)\left(\vec{q}_{1} \cdot \vec{\sigma}_{3 N}\right)+i \vec{\sigma}_{12} \cdot\left[\vec{q}_{1} \times \vec{n}\right]\right]\left(1+\vec{\tau}_{12} \cdot \vec{\tau}_{3 N}\right)\right.
\end{aligned}
$$


$\begin{array}{ll}+ & \left.i \vec{\sigma}_{3 N} \cdot\left[\vec{q}_{1} \times \vec{n}\right]\right]\left(3-\vec{\tau}_{12} \cdot \vec{\tau}_{3 N}\right) \\ + & \left.2\left[\left(\vec{q}_{2} \cdot \vec{\sigma}_{12}\right)\left(\vec{n} \cdot \vec{\sigma}_{3 N}\right)-\left(\vec{n} \cdot \vec{\sigma}_{12}\right)\left(\vec{q}_{2} \cdot \vec{\sigma}_{3 N}\right)+i \vec{\sigma}_{12} \cdot\left[\vec{q}_{2} \times \vec{n}\right]-i \vec{\sigma}_{3 N} \cdot\left[\vec{q}_{2} \times \vec{n}\right] \vec{\tau}_{12} \cdot \vec{\tau}_{3 N}\right]\right\} \\ - & \psi_{2}\left\{\left[\left(\vec{q}_{2} \cdot \vec{\sigma}_{12}\right)\left(\vec{n} \cdot \vec{\sigma}_{3 N}\right)-\left(\vec{n} \cdot \vec{\sigma}_{12}\right)\left(\vec{q}_{2} \cdot \vec{\sigma}_{3 N}\right)+i \vec{\sigma}_{12} \cdot\left[\vec{q}_{2} \times \vec{n}\right]\right]\left(1-\vec{\tau}_{12} \cdot \vec{\tau}_{3 N}\right)\right. \\ - & i \vec{\sigma}_{3 N} \cdot\left[\vec{q}_{2} \times \vec{n}\right]\left(3+\vec{\tau}_{12} \cdot \vec{\tau}_{3 N}\right) \\ + & \left.2\left[\left(\vec{q}_{1} \cdot \vec{\sigma}_{12}\right)\left(\vec{n} \cdot \vec{\sigma}_{3 N}\right)-\left(\vec{n} \cdot \vec{\sigma}_{12}\right)\left(\vec{q}_{1} \cdot \vec{\sigma}_{3 N}\right)+i \vec{\sigma}_{12} \cdot\left[\vec{q}_{1} \times \vec{n}\right]-i \vec{\sigma}_{3 N} \cdot\left[\vec{q}_{1} \times \vec{n}\right] \vec{\tau}_{12} \cdot \vec{\tau}_{3 N}\right]\right\}\end{array}$

$$
\begin{aligned}
& 4 \rightarrow 11: \quad\left(f_{1}, f_{2}\right) \otimes\left(\Sigma_{1}, \Sigma_{2}\right) \stackrel{(\sqrt{29}, c)}{\longrightarrow}\left(\varphi_{1}, \varphi_{2}\right)\left|\otimes\left(\chi_{1}, \chi_{2}\right) \stackrel{(197)}{\longrightarrow} \phi_{A}\right| \times \psi_{A} \stackrel{(40)}{\longrightarrow} \stackrel{(138)}{\Psi_{S}} \\
& \Psi_{S}=\psi_{A}\left\{-\left[\left(\vec{q}_{1} \cdot \vec{\sigma}_{12}\right)\left(\vec{n} \cdot \vec{\sigma}_{3 N}\right)-\left(\vec{n} \cdot \vec{\sigma}_{12}\right)\left(\vec{q}_{1} \cdot \vec{\sigma}_{3 N}\right)+i \vec{\sigma}_{12} \cdot\left[\vec{q}_{1} \times \vec{n}\right]\right]\left(3-\vec{\tau}_{12} \cdot \vec{\tau}_{3 N}\right)\right. \\
& -\quad\left[\left(\vec{q}_{2} \cdot \vec{\sigma}_{12}\right)\left(\vec{n} \cdot \vec{\sigma}_{3 N}\right)-\left(\vec{n} \cdot \vec{\sigma}_{12}\right)\left(\vec{q}_{2} \cdot \vec{\sigma}_{3 N}\right)+i \vec{\sigma}_{12} \cdot\left[\vec{q}_{2} \times \vec{n}\right]\right]\left(3+\vec{\tau}_{12} \cdot \vec{\tau}_{3 N}\right) \\
& \left.-\quad 3 i \vec{\sigma}_{3 N} \cdot\left[\vec{q}_{1} \times \vec{n}\right]\left(1+\vec{\tau}_{12} \cdot \vec{\tau}_{3 N}\right)+3 i \vec{\sigma}_{3 N} \cdot\left[\vec{q}_{2} \times \vec{n}\right]\left(1-\vec{\tau}_{12} \cdot \vec{\tau}_{3 N}\right)\right\}
\end{aligned}
$$$$
5 \rightarrow 10: \quad\left(f_{1}, f_{2}\right) \otimes\left(\Sigma_{1}, \Sigma_{2}\right) \stackrel{(89)}{\longrightarrow} \varphi_{S}\left|\times\left(\chi_{1}, \chi_{2}\right) \stackrel{(46)}{\longrightarrow}\left(\phi_{1}, \phi_{2}\right)\right| \times\left(\psi_{1}, \psi_{2}\right) \stackrel{(11)}{\longrightarrow} \stackrel{(139)}{\Psi_{S}}
$$$$
\Psi_{S}=-\psi_{1}\left\{\left(\vec{q}_{1} \cdot \vec{\sigma}_{12}\right)\left(\vec{n} \cdot \vec{\sigma}_{3 N}\right)-\left(\vec{n} \cdot \vec{\sigma}_{12}\right)\left(\vec{q}_{1} \cdot \vec{\sigma}_{3 N}\right)+\left(\vec{q}_{2} \cdot \vec{\sigma}_{12}\right)\left(\vec{n} \cdot \vec{\sigma}_{3 N}\right)-\left(\vec{n} \cdot \vec{\sigma}_{12}\right)\left(\vec{q}_{2} \cdot \vec{\sigma}_{3 N}\right)\right.
$$$$
\left.+\quad i \vec{\sigma}_{12} \cdot\left[\vec{q}_{1} \times \vec{n}\right]-i \vec{\sigma}_{3 N} \cdot\left[\vec{q}_{1} \times \vec{n}\right]+i \vec{\sigma}_{12} \cdot\left[\vec{q}_{2} \times \vec{n}\right]+i \vec{\sigma}_{3 N} \cdot\left[\vec{q}_{2} \times \vec{n}\right]\right\}\left(3-\vec{\tau}_{12} \cdot \vec{\tau}_{3 N}\right)
$$$$
+\psi_{2}\left\{\left(\vec{q}_{1} \cdot \vec{\sigma}_{12}\right)\left(\vec{n} \cdot \vec{\sigma}_{3 N}\right)-\left(\vec{n} \cdot \vec{\sigma}_{12}\right)\left(\vec{q}_{1} \cdot \vec{\sigma}_{3 N}\right)+\left(\vec{q}_{2} \cdot \vec{\sigma}_{12}\right)\left(\vec{n} \cdot \vec{\sigma}_{3 N}\right)-\left(\vec{n} \cdot \vec{\sigma}_{12}\right)\left(\vec{q}_{2} \cdot \vec{\sigma}_{3 N}\right)\right.
$$$$
\left.+\quad i \vec{\sigma}_{12} \cdot\left[\vec{q}_{1} \times \vec{n}\right]-i \vec{\sigma}_{3 N} \cdot\left[\vec{q}_{1} \times \vec{n}\right]+i \vec{\sigma}_{12} \cdot\left[\vec{q}_{2} \times \vec{n}\right]+i \vec{\sigma}_{3 N} \cdot\left[\vec{q}_{2} \times \vec{n}\right]\right\}\left(3+\vec{\tau}_{12} \cdot \vec{\tau}_{3 N}\right)
$$

$$
\begin{aligned}
& 6 \rightarrow 8: \quad\left(f_{1}, f_{2}\right) \times \stackrel{(79)}{\Sigma_{S}} \stackrel{(82, a)}{\longrightarrow}\left(\varphi_{1}, \varphi_{2}\right)\left|\otimes\left(\chi_{1}, \chi_{2}\right) \stackrel{(36)}{\longrightarrow} \phi_{S}\right| \times \psi_{S} \stackrel{\sqrt[39]{39}}{\longrightarrow} \stackrel{(140)}{\Psi_{S}} \\
& \Psi_{S}=\psi_{S}\left\{-\left[\left(\vec{q}_{1} \cdot \vec{\sigma}_{12}\right)\left(\vec{n} \cdot \vec{\sigma}_{3 N}\right)-\left(\vec{n} \cdot \vec{\sigma}_{12}\right)\left(\vec{q}_{1} \cdot \vec{\sigma}_{3 N}\right)-2 i \vec{\sigma}_{12} \cdot\left[\vec{q}_{1} \times \vec{n}\right]\right]\left(1-\vec{\tau}_{12} \cdot \vec{\tau}_{3 N}\right)\right. \\
& \left.+\quad\left[\left(\vec{q}_{2} \cdot \vec{\sigma}_{12}\right)\left(\vec{n} \cdot \vec{\sigma}_{3 N}\right)-\left(\vec{n} \cdot \vec{\sigma}_{12}\right)\left(\vec{q}_{2} \cdot \vec{\sigma}_{3 N}\right)-2 i \vec{\sigma}_{12} \cdot\left[\vec{q}_{2} \times \vec{n}\right]\right]\left(1+\vec{\tau}_{12} \cdot \vec{\tau}_{3 N}\right)\right\}
\end{aligned}
$$

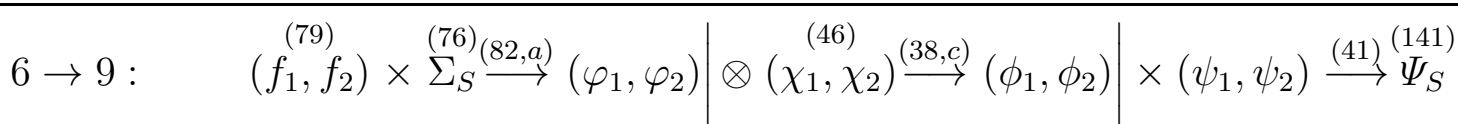

$$
\begin{aligned}
& \Psi_{S}=-\psi_{1}\left\{2\left[\left(\vec{q}_{1} \cdot \vec{\sigma}_{12}\right)\left(\vec{n} \cdot \vec{\sigma}_{3 N}\right)-\left(\vec{n} \cdot \vec{\sigma}_{12}\right)\left(\vec{q}_{1} \cdot \vec{\sigma}_{3 N}\right)-2 i \vec{\sigma}_{12} \cdot\left[\vec{q}_{1} \times \vec{n}\right]\right]\right. \\
& \left.+\quad+\left[\left(\vec{q}_{2} \cdot \vec{\sigma}_{12}\right)\left(\vec{n} \cdot \vec{\sigma}_{3 N}\right)-\left(\vec{n} \cdot \vec{\sigma}_{12}\right)\left(\vec{q}_{2} \cdot \vec{\sigma}_{3 N}\right)-2 i \vec{\sigma}_{12} \cdot\left[\vec{q}_{2} \times \vec{n}\right]\right]\left(1+\vec{\tau}_{12} \cdot \vec{\tau}_{3 N}\right)\right\} \\
& +\quad \psi_{2}\left\{\left[\left(\vec{q}_{1} \cdot \vec{\sigma}_{12}\right)\left(\vec{n} \cdot \vec{\sigma}_{3 N}\right)-\left(\vec{n} \cdot \vec{\sigma}_{12}\right)\left(\vec{q}_{1} \cdot \vec{\sigma}_{3 N}\right)-2 i \vec{\sigma}_{12} \cdot\left[\vec{q}_{1} \times \vec{n}\right]\right]\left(1-\vec{\tau}_{12} \cdot \vec{\tau}_{3 N}\right)\right. \\
& \left.+\quad+2\left[\left(\vec{q}_{2} \cdot \vec{\sigma}_{12}\right)\left(\vec{n} \cdot \vec{\sigma}_{3 N}\right)-\left(\vec{n} \cdot \vec{\sigma}_{12}\right)\left(\vec{q}_{2} \cdot \vec{\sigma}_{3 N}\right)-2 i \vec{\sigma}_{12} \cdot\left[\vec{q}_{2} \times \vec{n}\right]\right]\right\}
\end{aligned}
$$

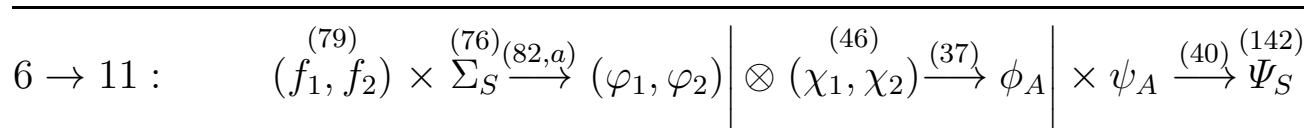

$$
\begin{aligned}
& \Psi_{S}=\psi_{A}\left\{\left[\left(\vec{q}_{1} \cdot \vec{\sigma}_{12}\right)\left(\vec{n} \cdot \vec{\sigma}_{3 N}\right)-\left(\vec{n} \cdot \vec{\sigma}_{12}\right)\left(\vec{q}_{1} \cdot \vec{\sigma}_{3 N}\right)-2 i \vec{\sigma}_{12} \cdot\left[\vec{q}_{1} \times \vec{n}\right]\right]\left(3+\vec{\tau}_{12} \cdot \vec{\tau}_{3 N}\right)\right. \\
& \left.+\quad\left[\left(\vec{q}_{2} \cdot \vec{\sigma}_{12}\right)\left(\vec{n} \cdot \vec{\sigma}_{3 N}\right)-\left(\vec{n} \cdot \vec{\sigma}_{12}\right)\left(\vec{q}_{2} \cdot \vec{\sigma}_{3 N}\right)-2 i \vec{\sigma}_{12} \cdot\left[\vec{q}_{2} \times \vec{n}\right]\right]\left(3-\vec{\tau}_{12} \cdot \vec{\tau}_{3 N}\right)\right\}
\end{aligned}
$$

\section{References}


[1] V.B. Berestetsky and M.V. Terentyev, Yad. Fiz. 24 (1976) 1044 [Sov. J. Nucl. Phys. 24 (1977) 547].

[2] B.L.G. Bakker, L.A. Kondratyuk and M.V. Terentyev, Nucl. Phys. B158 (1979) 497.

[3] F.M. Lev, Fortschr. Phys. 31 (1983) 75

[4] S. Capstick and N. Isgur, Phys. Rev. D34 (1986) 2809

[5] H.J. Weber, Ann. Phys. (N.Y.) 177 (1987) 38;

[6] W. Konen and H.J. Weber, Phys. Rev. D41 (1992) 2201;

H.J. Weber and X. Xu, Nucl. Phys. A600 (1996) 461.

[7] M. Beyer, C. Kuhrts and H.J. Weber, Relativistic Spin-Flavor States in Light Front Dynamics, Preprint, 1998, Submitted to Ann. of Phys. (N.Y.).

[8] Z. Dziembowski, Phys. Rev. D37 (1988) 768

[9] P.L. Chung and F. Coester, Phys. Rev. D44 (1991) 229

[10] Bo-Qiang Ma, J. Phys. G 17 (1991) L53;

Bo-Qiang Ma and Qi-Ren Zhang, Z.Phys. C58 (1993) 479

[11] S.J. Brodsky, F. Schlumpf, Phys. Lett. B329 (1994) 111

[12] B.D. Keister, Phys. Rev. D49 (1994) 1500.

[13] F. Cardarelli, E. Pace, G. Salmè and S. Simula, Phys. Lett. B357 (1995) 267.

[14] B.D. Keister and W.N. Polyzou, In: Advances in Nuclear Physics, ed. J.W. Negele and E.W. Vogt, (Plenum Press, New York) 20 (1991) 225.

[15] F. Coester, Prog. in Part. and Nucl. Phys., 29 (1992) 1.

[16] V.M. Kolybasov, Nucl. Phys. 68 (1965) 8

[17] V.A. Karmanov, ZhETF, 71 (1976) 399 [transl.: JETP, 44 (1976) 210]

[18] J. Carbonell, B. Desplanques, V.A. Karmanov and J.-F. Mathiot, Explicitly covariant light-front dynamics and relativistic few-body systems, To be published in Phys. Reports, 1998

[19] V.A. Karmanov, ZhETF, 76 (1979) 1884 [JETP, 49 (1979) 954]

[20] Yu.M. Shirokov, Dokl. Akad. Nauk SSSR 99 (1954) 737

[21] H.J. Melosh, Phys. Rev. D9 (1974) 1095

[22] J. Carbonell and V.A. Karmanov, Nucl. Phys. A581 (1995) 625

[23] V.A. Karmanov and J.-F. Mathiot, Nucl. Phys. A602 (1996) 388 